\title{
Strong convergence results for the asymptotic behavior of the 3D-shell model
}

\author{
D. Chapelle, A. Collin \\ Inria Saclay Ile-de-France, Palaiseau, France \\ Journal of Elasticity, DOI:10.1007/s10659-013-9452-3
}

\begin{abstract}
We revisit the asymptotic convergence properties - with respect to the thickness parameter - of the earlier-proposed 3D-shell model. This shell model is very attractive for engineering applications, in particular due to the possibility of directly using a general 3D constitutive law in the corresponding finite element formulations. We establish strong convergence results for the 3D-shell model in the two main types of asymptotic regimes, namely, bending- and membrane-dominated behavior. This is an important achievement, as it completely substantiates the asymptotic consistency of the 3D-shell model with 3D linearized isotropic elasticity.
\end{abstract}

Keywords: Shells; 3D-shell model; Asymptotic analysis

Mathematics Subject Classification (2010): 74K25; 35B40

\section{Introduction}

The mechanical behavior of thin shells has been described and discussed since the early ages of structural mechanics, albeit only much more recently has the problem been mathematically addressed with the point of view of asymptotic analysis, see [11, 21, 8] and references therein. The major benefits of such asymptotic analyses - in which the convergence of sequences of solutions associated with decreasing thickness of the structure is investigated - have been to justify earlier-introduced limit models - in particular the so-called membrane and pure bending models - and to shed light on what type of convergence can be expected, with subtle accompanying features such as boundary layers, see, e.g., $[12,19]$.

Shell models are in essence surface-based, namely, they are described by equations posed on a surface, i.e., the midsurface of the structure, in general, see in particular [16] for a classical example of shell model. Via an adequate geometric description of this surface based on a chart that maps a two-dimensional (2D) reference domain to the actual surface immersed in the Euclidean space, the model equations can be expressed as partial differential equations (PDEs) posed in the 2D reference domain. Consequently, appropriate discretization procedures can be defined, in particular with finite elements [4]. However, such models and discretizations thereof are seldom used in engineering practice nowadays. A first type of reason for this lies in the difficulties associated with the geometric definition of the surface, not well-suited to geometries typically produced by computer-aided design (CAD) systems. A second limitation - probably even more important - of classical shell models in the engineering context pertains to the need of considering wide varieties of material properties generally characterized in three-dimensional (3D) continuum mechanics, whereas these shell models have "built-in" constitutive assumptions allowing little latitude for material variations. 
By contrast, so-called "general shell elements" [3] or "degenerated solid shell elements" [1] are finite elements designed for shell structures - albeit directly based on 3D variational formulations, hence they can be accordingly used with 3D constitutive equations, without any reference to a given continuous shell model. In fact, it was later shown in [7] - see also [8] that there exists an implicit shell model underlying these finite element methods. Furthermore, this underlying model was proven to be asymptotically consistent with $3 \mathrm{D}$ linearized isotropic elasticity, meaning that asymptotic analyses for this shell model and for 3D elasticity reveal the same limit models, whether the asymptotic behavior be of membrane- or bending-dominated type [6]. Nevertheless, general shell elements still feature a limitation regarding the material law, as they require the use of a "plane stress" assumption, which is not straightforward to implement in the case of non-linear stress-strain laws.

The 3D-shell model and corresponding finite elements as proposed in [9] were introduced to completely overcome any restriction in the use of 3D constitutive equations, namely, they are posed as a 3D variational formulation in which 3D material laws can be used without any prior transformation. In addition, these 3D-shell models and elements employ quadratic kinematical assumptions across the thickness - instead of essentially linear expressions of displacements for classical shell models and elements - which allows for a more accurate description of complex deformation fields. This model was also shown to be asymptotically consistent with 3D elasticity, albeit only based on weak convergence results [8]. We may also refer to [18, 20, 2] for earlier results in similar approaches applied to plate theory. In the present paper we revisit the convergence results of the continuous 3D-shell model, and we show that convergence also holds in the strong sense, again for the two main types of asymptotic behavior. We point out that our new results are obtained under the same assumptions as in $[9,8]$, albeit the difference lies in the improved techniques of proof.

In the next section, we recall the 3D-shell model formulation and properties, before proceeding to introduce the asymptotic setting and analyse the asymptotic convergence behavior in Section 3.

\section{Model description}

\subsection{Geometry}

We consider a thin three-dimensional (3D) volume assumed to be defined by a midsurface $\mathcal{S}$, and a thickness parameter $t$ that may vary over the extent of $\mathcal{S}$. The surface $\mathcal{S}$ is described by a mapping $\vec{\phi}$ defined over $\bar{\omega}$, with $\omega$ a domain of $\mathbb{R}^{2}$, and with values in the three-dimensional Euclidean space $\mathcal{E}$. Namely, we have $\mathcal{S}=\vec{\phi}(\bar{\omega})$, and we assume that $\vec{\phi}$ is as regular as needed, so that we can introduce and employ the usual concepts of differential geometry, for which we will only give a brief summary, and refer to [8] - see also [14,11] - for a more detailed exposition. We start with the so-called surface covariant basis

$$
\vec{a}_{\alpha}=\vec{\phi}_{, \alpha}=\frac{\partial \vec{\phi}}{\partial \xi^{\alpha}}\left(\xi^{1}, \xi^{2}\right), \quad\left(\xi^{1}, \xi^{2}\right) \in \bar{\omega} .
$$

Note that we employ the standard convention that Greek indices vary in $\{1,2\}$, whereas Latin indices used later will vary in $\{1,2,3\}$. We then assume that the vectors $\left(\vec{a}_{1}, \vec{a}_{2}\right)$ provide a well-defined basis of the plane tangential to the midsurface, with the condition

$$
\left\|\vec{a}_{1} \wedge \vec{a}_{2}\right\|>0, \quad \forall\left(\xi^{1}, \xi^{2}\right) \in \bar{\omega},
$$

and we can thus introduce the unit normal vector

$$
\vec{a}_{3}=\frac{\vec{a}_{1} \wedge \vec{a}_{2}}{\left\|\vec{a}_{1} \wedge \vec{a}_{2}\right\|} .
$$




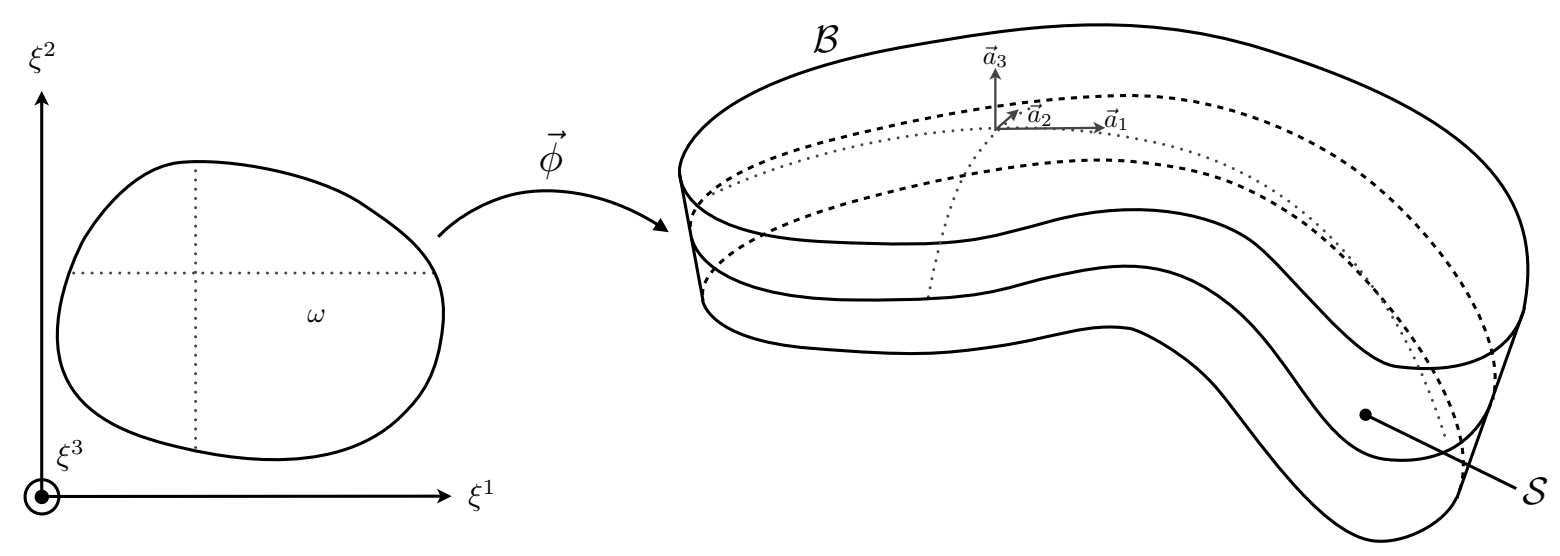

Figure 1: Geometrical description of thin 3D body

Alternatively, the contravariant basis - denoted with superscripts - is defined by

$$
\vec{a}^{\alpha} \cdot \vec{a}_{\beta}=\delta_{\beta}^{\alpha},
$$

where $\delta$ denotes the Kronecker symbol. The surface metric tensor - also called first fundamental form of the surface - is then associated with the components

$$
a_{\alpha \beta}=\vec{a}_{\alpha} \cdot \vec{a}_{\beta}, \quad a^{\alpha \beta}=\vec{a}^{\alpha} \cdot \vec{a}^{\beta},
$$

in covariant and contravariant forms, respectively. Intrinsic surface measures can be expressed as

$$
d S=\sqrt{a} d \xi^{1} d \xi^{2}, \quad \text { with } a=\left\|\vec{a}_{1} \wedge \vec{a}_{2}\right\|^{2}=a_{11} a_{22}-\left(a_{12}\right)^{2} .
$$

We next recall that the covariant components of the curvature tensor - also called second fundamental form - are given by

$$
b_{\alpha \beta}=\vec{a}_{3} \cdot \vec{a}_{\alpha, \beta},
$$

while the third fundamental form is defined by

$$
c_{\alpha \beta}=b_{\alpha}^{\lambda} b_{\lambda \beta}, \quad \text { with } b_{\alpha}^{\lambda}=a^{\lambda \mu} b_{\mu \alpha},
$$

where we use the Einstein convention of implicit summation for repeated indices - namely, for both $\lambda$ and $\mu$ here. Finally, we will need the concept of surface-based covariant differentiation of vectors, defined by

$$
u_{\alpha \mid \beta}=u_{\alpha, \beta}-\Gamma_{\alpha \beta}^{\lambda} u_{\lambda},
$$

where the comma denotes standard derivatives, and with the so-called surface Christoffel symbols given by

$$
\Gamma_{\alpha \beta}^{\lambda}=\vec{a}^{\lambda} \cdot \vec{a}_{\alpha, \beta}
$$

Defining now the 3D domain

$$
\Omega=\left\{\left(\xi^{1}, \xi^{2}, \xi^{3}\right) \in \mathbb{R}^{3} \mid\left(\xi^{1}, \xi^{2}\right) \in \omega, \xi^{3} \in\right]-\frac{t\left(\xi^{1}, \xi^{2}\right)}{2},+\frac{t\left(\xi^{1}, \xi^{2}\right)}{2}[\},
$$

the 3D geometry is described by the mapping $\vec{\Phi}$

$$
\vec{\Phi}\left(\xi^{1}, \xi^{2}, \xi^{3}\right)=\vec{\phi}\left(\xi^{1}, \xi^{2}\right)+\xi^{3} \vec{a}_{3}\left(\xi^{1}, \xi^{2}\right), \quad\left(\xi^{1}, \xi^{2}, \xi^{3}\right) \in \bar{\Omega},
$$


and the region of the Euclidean space occupied by the thin body is given by $\mathcal{B}=\vec{\Phi}(\bar{\Omega})$, see Figure 1. We will assume that the thickness does not degenerate anywhere, namely,

$$
t\left(\xi^{1}, \xi^{2}\right)>0, \quad \forall\left(\xi^{1}, \xi^{2}\right) \in \bar{\omega} .
$$

Accordingly, we can now define the $3 \mathrm{D}$ covariant basis

$$
\vec{g}_{i}=\vec{\Phi}_{, i}=\frac{\partial \vec{\Phi}}{\partial \xi^{i}}\left(\xi^{1}, \xi^{2}, \xi^{3}\right), \quad\left(\xi^{1}, \xi^{2}, \xi^{3}\right) \in \bar{\Omega}
$$

We infer $[8]$

$$
\vec{g}_{\alpha}=\left(\delta_{\alpha}^{\lambda}-\xi^{3} b_{\alpha}^{\lambda}\right) \vec{a}_{\lambda}, \quad \vec{g}_{3}=\vec{a}_{3},
$$

and the following covariant components for the $3 \mathrm{D}$ metric tensor $g_{i j}=\vec{g}_{i} \cdot \vec{g}_{j}$

$$
g_{\alpha \beta}=a_{\alpha \beta}-2 \xi^{3} b_{\alpha \beta}+\left(\xi^{3}\right)^{2} c_{\alpha \beta}, \quad g_{\alpha 3}=0, \quad g_{33}=1 .
$$

We will also use the contravariant 3D basis vectors such that

$$
\vec{g}^{i} \cdot \vec{g}_{j}=\delta_{j}^{i}
$$

and the contravariant components of the 3D metric tensor given by $g^{i j}=\vec{g}^{i} \cdot \vec{g}^{j}$. Finally, the volume measure is expressed as

$$
d V=\sqrt{g} d \xi^{1} d \xi^{2} d \xi^{3}
$$

with

$$
g=\left[\left(\vec{g}_{1} \wedge \vec{g}_{2}\right) \cdot \vec{g}_{3}\right]^{2}=a\left(1-2 H \xi^{3}+K\left(\xi^{3}\right)^{2}\right)^{2},
$$

where $H$ and $K$ respectively denote the mean and Gaussian curvatures of the surface. Note in passing that this shows that the condition $g>0$ necessary for the mapping $\vec{\Phi}$ to be well-defined is equivalent to $1-2 H \xi^{3}+K\left(\xi^{3}\right)^{2}>0$, namely also to

$$
t\left(\xi^{1}, \xi^{2}\right)<2\left|R_{\min }\left(\xi^{1}, \xi^{2}\right)\right|, \quad \forall\left(\xi^{1}, \xi^{2}\right) \in \bar{\omega},
$$

where $R_{\text {min }}$ denotes the radius of curvature of smallest absolute value at the specific point considered, condition that we will henceforth assume to be satisfied.

\subsection{D-shell model}

We start by recalling the formulation of linearized isotropic elasticity expressed for $\mathcal{B}$ using the above-introduced curvilinear coordinates. The variational formulation reads

$$
A^{3 D}(\vec{U}, \vec{V})=F^{3 D}(\vec{V}),
$$

for any $\vec{V}$ in a space of suitable test functions satisfying adequate boundary conditions, with

$$
A^{3 D}(\vec{U}, \vec{V})=\int_{\Omega} H^{i j k l} e_{i j}(\vec{U}) e_{k l}(\vec{V}) d V, \quad F^{3 D}(\vec{V})=\int_{\Omega} \vec{F} \cdot \vec{V} d V .
$$

In these expressions, $\vec{F}$ represents the external loading - assumed to be volume-distributed here - while the linearized strain tensor appearing in $A^{3 D}$ is defined by

$$
e_{i j}(\vec{V})=\frac{1}{2}\left(\vec{V}_{, i} \cdot \vec{g}_{j}+\vec{V}_{, j} \cdot \vec{g}_{i}\right),
$$


and the fourth-order constitutive tensor by

$$
H^{i j k l}=L_{1} g^{i j} g^{k l}+L_{2}\left(g^{i k} g^{j l}+g^{i l} g^{j k}\right),
$$

with $L_{1}$ and $L_{2}$ the Lamé constants, i.e.,

$$
L_{1}=E \frac{\nu}{(1+\nu)(1-2 \nu)}, \quad L_{2}=\frac{E}{2(1+\nu)}
$$

if we classically denote Young's modulus and Poisson's ratio by $E$ and $\nu$, respectively. Note that this entails the simplifications

$$
\left\{\begin{array}{l}
H^{\alpha \beta \gamma 3}\left(=H^{\alpha \beta 3 \gamma}=H^{\gamma 3 \alpha \beta}=H^{3 \gamma \alpha \beta}\right)=0, \quad \forall \alpha, \beta, \gamma=1,2 \\
H^{\alpha 333}\left(=H^{3 \alpha 33}=H^{33 \alpha 3}=H^{333 \alpha}\right)=0, \quad \forall \alpha=1,2
\end{array}\right.
$$

The 3D-shell model is defined as the variational projection of the 3D model onto the space of displacement functions having quadratic expressions in the $\xi^{3}$-variable. Namely, defining

$$
\left\{\begin{array}{l}
A^{3 D s}(\vec{u}, \vec{\theta}, \vec{\varrho} ; \vec{v}, \vec{\eta}, \vec{\varsigma})=A^{3 D}\left(\vec{u}+\xi^{3} \vec{\theta}+\left(\xi^{3}\right)^{2} \vec{\varrho}, \vec{v}+\xi^{3} \vec{\eta}+\left(\xi^{3}\right)^{2} \vec{\varsigma}\right) \\
F^{3 D s}(\vec{v}, \vec{\eta}, \vec{\varsigma})=F^{3 D}\left(\vec{v}+\xi^{3} \vec{\eta}+\left(\xi^{3}\right)^{2} \vec{\varsigma}\right)
\end{array}\right.
$$

we consider the variational equation

$$
A^{3 D s}(\vec{u}, \vec{\theta}, \vec{\varrho} ; \vec{v}, \vec{\eta}, \vec{\varsigma})=F^{3 D s}(\vec{v}, \vec{\eta}, \vec{\varsigma}),
$$

see also $[13,5]$ for discussions on this type of kinematical assumption. With these specific displacement functions, we have the following identities for the linearized strains $[9,8]$

$$
\left\{\begin{array}{l}
e_{\alpha \beta}(\vec{U})=\gamma_{\alpha \beta}(\vec{u})+\xi^{3} \hat{\chi}_{\alpha \beta}(\vec{u}, \vec{\theta})+\left(\xi^{3}\right)^{2} k_{\alpha \beta}(\vec{\theta}, \vec{\varrho})+\left(\xi^{3}\right)^{3} l_{\alpha \beta}(\vec{\varrho}) \\
e_{\alpha 3}(\vec{U})=\zeta_{\alpha}(\vec{u}, \vec{\theta})+\xi^{3} m_{\alpha}(\vec{\theta}, \vec{\varrho})+\left(\xi^{3}\right)^{2} n_{\alpha}(\vec{\varrho}) \\
e_{33}(\vec{U})=\delta(\vec{\theta})+\xi^{3} p(\vec{\varrho})
\end{array}\right.
$$

where

$$
\left\{\begin{array}{l}
\gamma_{\alpha \beta}(\vec{u})=\frac{1}{2}\left(u_{\alpha \mid \beta}+u_{\beta \mid \alpha}\right)-b_{\alpha \beta} u_{3} \\
\hat{\chi}_{\alpha \beta}(\vec{u}, \vec{\theta})=\frac{1}{2}\left(\theta_{\alpha \mid \beta}+\theta_{\beta \mid \alpha}-b_{\alpha}^{\lambda} u_{\lambda \mid \beta}-b_{\beta}^{\lambda} u_{\lambda \mid \alpha}\right)-b_{\alpha \beta} \theta_{3}+c_{\alpha \beta} u_{3} \\
k_{\alpha \beta}(\vec{\theta}, \vec{\varrho})=\frac{1}{2}\left(\varrho_{\alpha \mid \beta}+\varrho_{\beta \mid \alpha}-b_{\alpha}^{\lambda} \theta_{\lambda \mid \beta}-b_{\beta}^{\lambda} \theta_{\lambda \mid \alpha}\right)-b_{\alpha \beta} \varrho_{3}+c_{\alpha \beta} \theta_{3} \\
l_{\alpha \beta}(\vec{\varrho})=-\frac{1}{2}\left(b_{\alpha}^{\lambda} \varrho_{\lambda \mid \beta}+b_{\beta}^{\lambda} \varrho_{\lambda \mid \alpha}\right)+c_{\alpha \beta} \varrho_{3} \\
\zeta_{\alpha}(\vec{u}, \vec{\theta})=\frac{1}{2}\left(\theta_{\alpha}+b_{\alpha}^{\lambda} u_{\lambda}+u_{3, \alpha}\right) \\
m_{\alpha}(\vec{\theta}, \vec{\varrho})=\frac{1}{2}\left(2 \varrho_{\alpha}+\theta_{3, \alpha}\right) \\
n_{\alpha}(\vec{\varrho})=\frac{1}{2}\left(-b_{\alpha}^{\lambda} \varrho_{\lambda}+\varrho_{3, \alpha}\right) \\
\delta(\vec{\theta})=\theta_{3} \\
p(\vec{\varrho})=2 \varrho_{3}
\end{array}\right.
$$

In our analyses we will use intrinsic Sobolev norms on $\mathcal{S}$ and $\mathcal{B}$, namely, integrating with surface and volume measures, respectively, and employing covariant derivatives whenever needed [8]. These norms are equivalent to the usual Sobolev norms defined on $\omega$ and $\Omega$, respectively, albeit they are intrinsic, i.e., they do not depend on the particular choice of coordinate system used to describe the actual geometry. In the sequel, we will also use the subscripts 0 and 1 to denote the intrinsic norms of $L^{2}(\mathcal{S})$ and $H^{1}(\mathcal{S})$, respectively.

Then, defining the space $\mathcal{V}^{3 D s}=\left[H^{1}(\mathcal{S}) \cap \mathcal{B C}\right]^{3}$ where $\mathcal{B C}$ symbolically denotes functions having vanishing trace on a part of non-zero measure of the boundary $\partial \omega$ - namely, Dirichlet (i.e. essential) boundary conditions - we recall the following result $[9,8]$, where we use the symbol $C$ to denote a generic strictly positive constant. 
Proposition 1 Assuming that $\vec{F} \in L^{2}(\mathcal{B})$, there is a unique $(\vec{u}, \vec{\theta}, \vec{\varrho})$ in $\mathcal{V}^{3 D s}$ that satisfies (13) for any $(\vec{v}, \vec{\eta}, \vec{\varsigma})$ in $\mathcal{V}^{3 D s}$, and we have

$$
\|\vec{u}, \vec{\theta}, \vec{\varrho}\|_{H^{1}(\mathcal{S})} \leq C\|\vec{F}\|_{L^{2}(\mathcal{B})} .
$$

\section{$3 \quad$ Asymptotic analysis}

\subsection{Asymptotic setting}

We now proceed to recast the above problem in an asymptotic framework. Denoting by $L$ an overall characteristic dimension of the surface, - e.g., the diameter - and

$$
t_{\min }=\inf _{\left(\xi^{1}, \xi^{2}\right) \in \omega} t\left(\xi^{1}, \xi^{2}\right)>0,
$$

we define the dimensionless thickness parameter

$$
\varepsilon=\frac{t_{\min }}{L}
$$

and

$$
l\left(\xi^{1}, \xi^{2}\right)=\frac{t\left(\xi^{1}, \xi^{2}\right)}{\varepsilon}=\frac{t\left(\xi^{1}, \xi^{2}\right)}{t_{\min }} L,
$$

hence $\inf _{\left(\xi^{1}, \xi^{2}\right) \in \omega} l\left(\xi^{1}, \xi^{2}\right)=L$, and we call $l$ the thickness profile. We then consider a sequence of shell geometries obtained by fixing the midsurface $\mathcal{S}$ and thickness profile $l$, and varying the $\varepsilon$ parameter. We subsequently seek a scaling of the loading in the form

$$
\vec{F}=\varepsilon^{\rho-1} \vec{G}
$$

with $\vec{G}$ independent of $\varepsilon$, and the parameter $\rho$ chosen so that the associated sequence of solutions $\left(\vec{u}^{\varepsilon}, \vec{\theta}^{\varepsilon}, \vec{\varrho}^{\varepsilon}\right) \in \mathcal{V}^{3 D s}$ satisfying

$$
A^{3 D s}\left(\vec{u}^{\varepsilon}, \vec{\theta}^{\varepsilon}, \vec{\varrho}^{\varepsilon} ; \vec{v}, \vec{\eta}, \vec{\varsigma}\right)=F^{3 D s}(\vec{v}, \vec{\eta}, \vec{\varsigma}), \quad \forall(\vec{v}, \vec{\eta}, \vec{\varsigma}) \in \mathcal{V}^{3 D s},
$$

has a non-zero finite limit energy-wise, namely, so that $\varepsilon^{-\rho} F^{3 D s}\left(\vec{u}^{\varepsilon}, \vec{\theta}^{\varepsilon}, \vec{\varrho}^{\varepsilon}\right)$ has a non-zero finite limit. Note that the expressions of $A^{3 D s}$ and $F^{3 D s}$ include a dependence on $\varepsilon$ even though this is not reflected in their notations for the sake of compactness. It can be shown that there is at most one value of this scaling parameter for which this convergence holds, and that such a value necessarily satisfies [8]

$$
1 \leq \rho \leq 3
$$

Several cases may then arise depending on the contents of the particular subspace

$$
\begin{aligned}
\mathcal{V}_{0}^{3 D s}=\{(\vec{v}, \vec{\eta}, \vec{\zeta}) & \in \mathcal{V}^{3 D s}, \text { such that } \\
\gamma_{\alpha \beta}(\vec{v}) & \left.=0, \zeta_{\alpha}(\vec{v}, \vec{\eta})=0, \delta(\vec{\eta})=0, \forall \alpha, \beta=1,2\right\},
\end{aligned}
$$

called the subspace of pure bending displacements. More specifically, we will distinguish two situations according to whether we have

$$
\mathcal{V}_{0}^{3 D s} \cap\left\{(\vec{v}, \vec{\eta}, \overrightarrow{0}) \in \mathcal{V}^{3 D s}\right\}=\{(\overrightarrow{0}, \overrightarrow{0}, \overrightarrow{0})\},
$$

in which case we say that pure bending is inhibited. By contrast, the situation when $\mathcal{V}_{0}^{3 D s}$ has non-trivial contents is referred to as non-inhibited pure bending. Both cases are frequently 
encountered in actual shells, depending on the surface geometry and on the boundary conditions considered, see [8] in particular.

Let us introduce some definitions and notation that will be needed in our asymptotic analysis. We will assume that the scaled loading $\vec{G}$ in (17) is of the form

$$
\vec{G}\left(\xi^{1}, \xi^{2}, \xi^{3}\right)=\vec{G}_{0}\left(\xi^{1}, \xi^{2}\right)+\xi^{3} \vec{B}\left(\xi^{1}, \xi^{2}, \xi^{3}\right),
$$

where $\vec{G}_{0}$ is in $L^{2}(\mathcal{S})$ and $\vec{B}$ is a bounded function over $\mathcal{B}$, uniformly in $\varepsilon$. We then define

$$
G^{3 D s}(\vec{v})=\int_{\omega} l \vec{G}_{0} \cdot \vec{v} d S .
$$

We now define the bilinear forms

$$
\begin{array}{r}
A_{\mathrm{m}}^{3 D s}(\vec{u}, \vec{\theta} ; \vec{v}, \vec{\eta})=\int_{\omega} l\left[{ }^{0} H^{\alpha \beta \lambda \mu} \gamma_{\alpha \beta}(\vec{u}) \gamma_{\lambda \mu}(\vec{v})+{ }^{0} H^{\alpha \beta 33}\left(\gamma_{\alpha \beta}(\vec{u}) \delta(\vec{\eta})+\gamma_{\alpha \beta}(\vec{v}) \delta(\vec{\theta})\right)\right. \\
\left.+4^{0} H^{\alpha 3 \beta 3} \zeta_{\alpha}(\vec{u}, \vec{\theta}) \zeta_{\beta}(\vec{v}, \vec{\eta})+{ }^{0} H^{3333} \delta(\vec{\theta}) \delta(\vec{\eta})\right] d S, \\
\begin{aligned}
A_{\mathrm{b}}^{3 D s}(\vec{u}, \vec{\theta}, \vec{\varrho} ; \vec{v}, \vec{\eta}, \vec{\varsigma}) & =\int_{\omega} \frac{l^{3}}{12}\left[{ }^{0} H^{\alpha \beta \lambda \mu} \hat{\chi}_{\alpha \beta}(\vec{u}, \vec{\theta}) \hat{\chi} \lambda \mu(\vec{v}, \vec{\eta})+{ }^{0} H^{\alpha \beta 33}\left(\hat{\chi}_{\alpha \beta}(\vec{u}, \vec{\theta}) p(\vec{\varsigma})\right.\right. \\
& \left.\left.+\hat{\chi}_{\alpha \beta}(\vec{v}, \vec{\eta}) p(\vec{\varrho})\right)+4^{0} H^{\alpha 3 \beta 3} m_{\alpha}(\vec{\theta}, \vec{\varrho}) m_{\beta}(\vec{\eta}, \vec{\varsigma})+{ }^{0} H^{3333} p(\vec{\varrho}) p(\vec{\varsigma})\right] d S,
\end{aligned}
\end{array}
$$

where

$$
{ }^{0} H^{i j k l}=\left.H^{i j k l}\right|_{\xi^{3}=0} .
$$

We will also use the following expansion of this constitutive tensor

$$
H^{i j k l}\left(\xi^{1}, \xi^{2}, \xi^{3}\right)={ }^{0} H^{i j k l}\left(\xi^{1}, \xi^{2}\right)+\xi^{3} \bar{H}^{i j k l}\left(\xi^{1}, \xi^{2}, \xi^{3}\right),
$$

with $\bar{H}^{i j k l}\left(\xi^{1}, \xi^{2}, \xi^{3}\right)$ bounded over $\mathcal{B}$ since the geometry is smooth.

Let us introduce the norm

$$
\|\vec{v}, \vec{\eta}, \vec{\varsigma}\|_{\mathrm{b}}=\left(\|\vec{v}\|_{1}^{2}+\|\underline{\eta}\|_{1}^{2}+\left\|\eta_{3}\right\|_{0}^{2}+\left\|\varsigma_{3}\right\|_{0}^{2}+\left\|\underline{\varsigma}+\frac{1}{2} \underline{\nabla} \eta_{3}\right\|_{0}^{2}\right)^{\frac{1}{2}},
$$

and the semi-norm

$$
\|\vec{v}, \vec{\eta}\|_{\mathrm{m}}=A_{\mathrm{m}}^{3 D s}(\vec{v}, \vec{\eta} ; \vec{v}, \vec{\eta})^{\frac{1}{2}} .
$$

Note that we classically use the underbar notation to denote tensors attached to the tangential plane of the surface $\mathcal{S}$, namely with component indices ranging in $\{1,2\}-$ e.g. first-order tensors correspond to vectors lying in the tangential plane, second-order tensors to linear mappings within this vector space, and so on - see [8] for more detail. As we will see in the following lemma, when pure bending is inhibited $\|\cdot\|_{\mathrm{m}}$ is a norm. In this lemma we recall some equivalence properties for the above norms or semi-norms - established in [8] in a straightforward manner - in which we dispense with indicating the (obvious) operands of strain tensors to alleviate the notation.

Lemma 1 We have an equivalence of norms - or semi-norms, when applicable - between:

1. $\|\vec{v}, \vec{\eta}\|_{\mathrm{m}}$ and $\left(\|\underline{\underline{\gamma}}\|_{0}^{2}+\|\underline{\zeta}\|_{0}^{2}+\|\delta\|_{0}^{2}\right)^{1 / 2} ;$

2. $\|\vec{v}, \vec{\eta}, \vec{\varsigma}\|_{\mathrm{b}}$ and $\left(\|\underline{\underline{\gamma}}\|_{0}^{2}+\|\underline{\zeta}\|_{0}^{2}+\|\delta\|_{0}^{2}+\|\underline{\underline{\hat{\chi}}}\|_{0}^{2}+\|\underline{m}\|_{0}^{2}+\|p\|_{0}^{2}\right)^{\frac{1}{2}}$; 
3. $\|\vec{v}, \vec{\eta}, \vec{\varsigma}\|_{1}$ and $\left(\|\underline{\underline{\gamma}}\|_{0}^{2}+\|\underline{\zeta}\|_{0}^{2}+\|\delta\|_{0}+\|\underline{\underline{\hat{\chi}}}\|_{0}^{2}+\|\underline{\underline{m}}\|_{0}^{2}+\|p\|_{0}^{2}+\|\underline{\underline{k}}\|_{0}^{2}+\|\underline{n}\|_{0}^{2}\right)^{\frac{1}{2}}$.

Note that none of the norm - or semi-norm - expressions used in this lemma depends on $\varepsilon$, hence all these equivalence properties hold independently of $\varepsilon$.

In our below asymptotic analyses we will use a decomposition of $A^{3 D s}$ obtained by using (12), (14), and the change of variables $\xi^{3}=\varepsilon \xi$, viz.

$$
A^{3 D s}(\vec{u}, \vec{\theta}, \vec{\varrho} ; \vec{v}, \vec{\eta}, \vec{\varsigma})=I_{1}+I_{2}+I_{3}+I_{4}+I_{5},
$$

with

$$
\begin{aligned}
& I_{1}(\vec{U}, \vec{V})=\varepsilon \int_{\omega} \int_{-l / 2}^{l / 2} H^{\alpha \beta \lambda \mu}\left[\gamma_{\alpha \beta}(\vec{u})+\varepsilon \xi \hat{\chi}_{\alpha \beta}(\vec{u}, \vec{\theta})+\varepsilon^{2}(\xi)^{2} k_{\alpha \beta}(\vec{\theta}, \vec{\varrho})+\varepsilon^{3}(\xi)^{3} l_{\alpha \beta}(\vec{\varrho})\right] \\
& \times\left[\gamma_{\lambda \mu}(\vec{v})+\varepsilon \xi \hat{\chi}_{\lambda \mu}(\vec{v}, \vec{\eta})+\varepsilon^{2}(\xi)^{2} k_{\lambda \mu}(\vec{\eta}, \vec{\varsigma})+\varepsilon^{3}(\xi)^{3} l_{\lambda \mu}(\vec{\varsigma})\right] \sqrt{g} d \xi d \xi^{1} d \xi^{2}, \\
& I_{2}(\vec{U}, \vec{V})=\varepsilon \int_{\omega} \int_{-l / 2}^{l / 2} 4 H^{\alpha 3 \beta 3}\left[\zeta_{\alpha}(\vec{u}, \vec{\theta})+\varepsilon \xi m_{\alpha}(\vec{\theta}, \vec{\varrho})+\varepsilon^{2}(\xi)^{2} n_{\alpha}(\vec{\varrho})\right] \\
& \times\left[\zeta_{\beta}(\vec{v}, \vec{\eta})+\varepsilon \xi m_{\beta}(\vec{\eta}, \vec{\varsigma})+\varepsilon^{2}(\xi)^{2} n_{\beta}(\vec{\varsigma})\right] \sqrt{g} d \xi d \xi^{1} d \xi^{2}, \\
& I_{3}(\vec{U}, \vec{V})=\varepsilon \int_{\omega} \int_{-l / 2}^{l / 2} H^{3333}[\delta(\vec{\theta})+\varepsilon \xi p(\vec{\varrho})] \times[\delta(\vec{\eta})+\varepsilon \xi p(\vec{\varsigma})] \sqrt{g} d \xi d \xi^{1} d \xi^{2}, \\
& I_{4}(\vec{U}, \vec{V})=\varepsilon \int_{\omega} \int_{-l / 2}^{l / 2} H^{\alpha \beta 33}\left[\gamma_{\alpha \beta}(\vec{u})+\varepsilon \xi \hat{\chi}_{\alpha \beta}(\vec{u}, \vec{\theta})+\varepsilon^{2}(\xi)^{2} k_{\alpha \beta}(\vec{\theta}, \vec{\varrho})+\varepsilon^{3}(\xi)^{3} l_{\alpha \beta}(\vec{\varrho})\right] \\
& \times[\delta(\vec{\eta})+\varepsilon \xi p(\vec{\varsigma})] \sqrt{g} d \xi d \xi^{1} d \xi^{2}, \\
& I_{5}(\vec{U}, \vec{V})=\varepsilon \int_{\omega} \int_{-l / 2}^{l / 2} H^{\alpha \beta 33}\left[\gamma_{\alpha \beta}(\vec{v})+\varepsilon \xi \hat{\chi}_{\alpha \beta}(\vec{v}, \vec{\eta})+\varepsilon^{2}(\xi)^{2} k_{\alpha \beta}(\vec{\eta}, \vec{\varsigma})+\varepsilon^{3}(\xi)^{3} l_{\alpha \beta}(\vec{\varsigma})\right] \\
& \times[\delta(\vec{\theta})+\varepsilon \xi p(\vec{\varrho})] \sqrt{g} d \xi d \xi^{1} d \xi^{2},
\end{aligned}
$$

using the compact notation

$$
\vec{U}=\vec{u}+\xi^{3} \vec{\theta}+\left(\xi^{3}\right)^{2} \vec{\varrho}, \quad \vec{V}=\vec{v}+\xi^{3} \vec{\eta}+\left(\xi^{3}\right)^{2} \vec{\varsigma} .
$$

Similarly, the linear form $F^{3 D s}$ gives

$$
F^{3 D s}(\vec{v}, \vec{\eta}, \vec{\varsigma})=\varepsilon \int_{\omega} \int_{-l / 2}^{l / 2} \vec{F} \cdot\left[\vec{v}+\varepsilon \xi \vec{\eta}+\varepsilon^{2}(\xi)^{2} \vec{\varsigma}\right] \sqrt{g} d \xi d \xi^{1} d \xi^{2}
$$

Finally, we will henceforth denote by $C$ generic constants independent of $\varepsilon$, and by $R_{\varepsilon}$ scalar quantities that tend to zero with $\varepsilon$. 


\subsection{Non-inhibited pure bending}

We start with the case of non-inhibited pure bending, namely, when

$$
\mathcal{V}_{0}^{3 D s} \cap\left\{(\vec{v}, \vec{\eta}, \overrightarrow{0}) \in \mathcal{V}^{3 D s}\right\} \neq\{(\overrightarrow{0}, \overrightarrow{0}, \overrightarrow{0})\} .
$$

In this case, as in more standard shell models the expected asymptotic behavior is that of a penalization mechanism in which the solution "tends to belong" to the constrained subspace $\mathcal{V}_{0}^{3 D s}$, associated with a scaling parameter $\rho=3$, see [8] and references therein.

We then define $\mathcal{V}_{\mathrm{b}}^{3 D s}$ as the completion of $\mathcal{V}^{3 D s}$ for the norm $\|\cdot\|_{\mathrm{b}}$. We will also use the space $\mathcal{V}_{0 \mathrm{~b}}^{3 D s}$, defined as the completion of $\mathcal{V}_{0}^{3 D s}$ for $\|\cdot\|_{\mathrm{b}}$, which is identified as

$$
\begin{aligned}
& \mathcal{V}_{0 \mathrm{~b}}^{3 D s}=\left\{(\vec{v}, \vec{\eta}, \vec{\varsigma}) \in \mathcal{V}_{\mathrm{b}}^{3 D s} \text { such that } \gamma_{\alpha \beta}(\vec{v})=0,\right. \\
& \left.\qquad \zeta_{\alpha}(\vec{v}, \vec{\eta})=0, \delta(\vec{\eta})=0, \forall \alpha, \beta=1,2\right\} .
\end{aligned}
$$

Using the proposed scaling $\rho=3$, the tentative limit problem reads:

Find $\left(\vec{u}^{0}, \vec{\theta}^{0}, \vec{\varrho}^{0}\right) \in \mathcal{V}_{0 \mathrm{~b}}^{3 D s}$ such that

$$
A_{\mathrm{b}}^{3 D s}\left(\vec{u}^{0}, \vec{\theta}^{0}, \vec{\varrho}^{0} ; \vec{v}, \vec{\eta}, \vec{\varsigma}\right)=G^{3 D s}(\vec{v}), \quad \forall(\vec{v}, \vec{\eta}, \vec{\varsigma}) \in \mathcal{V}_{0 \mathrm{~b}}^{3 D s} .
$$

Note that the right-hand side of this variational formulation defines a linear form in $\mathcal{V}_{0 \mathrm{~b}}^{3 D s}$ (although this completed space may contain elements that do not belong to $\mathcal{V}^{3 D s}$ ) since

$$
\begin{aligned}
\left|\int_{\omega} l \vec{G}_{0} \cdot \vec{v} d S\right| & \leq C\left\|\vec{G}_{0}\right\|_{0}\|\vec{v}\|_{0} \\
& \leq C\left\|\vec{G}_{0}\right\|_{0}\|\vec{v}, \vec{\eta}, \vec{\varsigma}\|_{\mathrm{b}}, \forall(\vec{v}, \vec{\eta}, \vec{\varsigma}) \in \mathcal{V}_{\mathrm{b}}^{3 D s}
\end{aligned}
$$

hence this variational problem is well-posed by construction. In this framework we have the following weak convergence $[9,8]$.

Lemma 2 The solution $\left(\vec{u}^{\varepsilon}, \vec{\theta}^{\varepsilon}, \vec{\varrho}^{\varepsilon}\right)$ of Problem (18) converges weakly in $\mathcal{V}_{\mathrm{b}}^{3 D s}$, as $\varepsilon$ goes to 0 , to $\left(\vec{u}^{0}, \vec{\theta}^{0}, \vec{\varrho}^{0}\right) \in \mathcal{V}_{0 \mathrm{~b}}^{3 D s}$ solution of $(36)$.

We proceed to establish the corresponding strong convergence, for which we will use the following uniform bound obtained in the proof of Lemma 2

$$
\frac{1}{\varepsilon}\left\|\vec{u}^{\varepsilon}, \vec{\theta}^{\varepsilon}\right\|_{\mathrm{m}}+\left\|\vec{u}^{\varepsilon}, \vec{\theta}^{\varepsilon}, \vec{\varrho}^{\varepsilon}\right\|_{\mathrm{b}}+\varepsilon\left\|\vec{u}^{\varepsilon}, \vec{\theta}^{\varepsilon}, \vec{\varrho}^{\varepsilon}\right\|_{1} \leq C .
$$

Proposition 2 The solution $\left(\vec{u}^{\varepsilon}, \vec{\theta}^{\varepsilon}, \vec{\varrho}^{\varepsilon}\right)$ of Problem (18) converges strongly in $\mathcal{V}_{\mathrm{b}}^{3 D s}$ to $\left(\vec{u}^{0}, \vec{\theta}^{0}, \vec{\varrho}^{0}\right) \in$ $\mathcal{V}_{0 \mathrm{~b}}^{3 D}$ solution of (36), as $\varepsilon$ goes to 0 . Moreover, we have that $\frac{1}{\varepsilon}\left\|\vec{u}^{\varepsilon}, \vec{\theta}^{\varepsilon}\right\|_{\mathrm{m}}$ and $\varepsilon\left\|\vec{u}^{\varepsilon}, \vec{\theta}^{\varepsilon}, \vec{\varrho}^{\varepsilon}\right\|_{1}$ both tend to zero.

Proof Considering the decomposition (29) we first have

$$
\begin{aligned}
& I_{1}\left(\vec{U}^{\varepsilon}, \vec{U}^{\varepsilon}\right)=\varepsilon \int_{\omega} l^{0} H^{\alpha \beta \lambda \mu}\left(\gamma_{\alpha \beta}\left(\vec{u}^{\varepsilon}\right) \gamma_{\lambda \mu}\left(\vec{u}^{\varepsilon}\right)+\frac{t^{2}}{6} \gamma_{\alpha \beta}\left(\vec{u}^{\varepsilon}\right) k_{\lambda \mu}\left(\vec{\theta}^{\varepsilon}, \vec{\varrho}^{\varepsilon}\right)\right. \\
& \left.\quad+\frac{t^{2}}{12} \hat{\chi}_{\alpha \beta}\left(\vec{u}^{\varepsilon}, \vec{\theta}^{\varepsilon}\right) \hat{\chi}_{\lambda \mu}\left(\vec{u}^{\varepsilon}, \vec{\theta}^{\varepsilon}\right)+\frac{t^{4}}{80} k_{\alpha \beta}\left(\vec{\theta}^{\varepsilon}, \vec{\varrho}^{\varepsilon}\right) k_{\lambda \mu}\left(\vec{\theta}^{\varepsilon}, \vec{\varrho}^{\varepsilon}\right)\right) \sqrt{a} d \xi^{1} d \xi^{2}+\bar{I}_{1}\left(\vec{U}^{\varepsilon}, \vec{U}^{\varepsilon}\right) \\
& \quad={ }^{0} I_{1}\left(\vec{U}^{\varepsilon}, \vec{U}^{\varepsilon}\right)+\bar{I}_{1}\left(\vec{U}^{\varepsilon}, \vec{U}^{\varepsilon}\right),
\end{aligned}
$$

in which we can show that $\frac{1}{\varepsilon^{3}} \bar{I}_{1}\left(\vec{U}^{\varepsilon}, \vec{U}^{\varepsilon}\right)=R_{\varepsilon}$ using (38) and Lemma 1. Defining next

$$
D_{1, \varepsilon}=\int_{\omega} \frac{l^{3}}{12}{ }^{0} H^{\alpha \beta \lambda \mu} \hat{\chi}_{\alpha \beta}\left(\vec{u}^{\varepsilon}-\vec{u}^{0}, \vec{\theta}^{\varepsilon}-\vec{\theta}^{0}\right) \hat{\chi}_{\lambda \mu}\left(\vec{u}^{\varepsilon}-\vec{u}^{0}, \vec{\theta}^{\varepsilon}-\vec{\theta}^{0}\right) d S,
$$


and

$$
D_{1, \varepsilon}^{\prime}=\int_{\omega} \frac{l^{3}}{12}{ }^{0} H^{\alpha \beta \lambda \mu} \hat{\chi}_{\alpha \beta}\left(\vec{u}^{\varepsilon}, \vec{\theta}^{\varepsilon}\right) \hat{\chi}_{\lambda \mu}\left(\vec{u}^{\varepsilon}, \vec{\theta}^{\varepsilon}\right) d S,
$$

we develop $D_{1, \varepsilon}$, and by using Lemma 2 we obtain

$$
\begin{aligned}
D_{1, \varepsilon} & =D_{1, \varepsilon}^{\prime}+\int_{\omega} \frac{l^{3}}{12}{ }^{0} H^{\alpha \beta \lambda \mu}\left(\hat{\chi}_{\alpha \beta}\left(\vec{u}^{0}, \vec{\theta}^{0}\right) \hat{\chi}_{\lambda \mu}\left(\vec{u}^{0}, \vec{\theta}^{0}\right)-2 \hat{\chi}_{\alpha \beta}\left(\vec{u}^{\varepsilon}, \vec{\theta}^{\varepsilon}\right) \hat{\chi}_{\lambda \mu}\left(\vec{u}^{0}, \vec{\theta}^{0}\right) d S\right) \\
& =D_{1, \varepsilon}^{\prime}-\int_{\omega} \frac{l^{3}}{12}{ }^{0} H^{\alpha \beta \lambda \mu} \hat{\chi}_{\alpha \beta}\left(\vec{u}^{0}, \vec{\theta}^{0}\right) \hat{\chi}_{\lambda \mu}\left(\vec{u}^{0}, \vec{\theta}^{0}\right) d S+R_{\varepsilon},
\end{aligned}
$$

with

$$
\begin{aligned}
D_{1, \varepsilon}^{\prime} & =\frac{1}{\varepsilon^{3}} I_{1}\left(\vec{U}^{\varepsilon}, \vec{U}^{\varepsilon}\right)-\frac{1}{\varepsilon^{2}} \int_{\omega} l^{0} H^{\alpha \beta \lambda \mu} \gamma_{\alpha \beta}\left(\vec{u}^{\varepsilon}\right) \gamma_{\lambda \mu}\left(\vec{u}^{\varepsilon}\right) d S \\
& -\int_{\omega} \frac{l^{3}}{6} H^{\alpha \beta \lambda \mu} \gamma_{\alpha \beta}\left(\vec{u}^{\varepsilon}\right) k_{\lambda \mu}\left(\vec{\theta}^{\varepsilon}, \vec{\varrho}^{\varepsilon}\right) d S-\varepsilon^{2} \int_{\omega} \frac{l^{5}}{80}{ }^{0} H^{\alpha \beta \lambda \mu} k_{\alpha \beta}\left(\vec{\theta}^{\varepsilon}, \vec{\varrho}^{\varepsilon}\right) k_{\lambda \mu}\left(\vec{\theta}^{\varepsilon}, \vec{\varrho}^{\varepsilon}\right) d S .
\end{aligned}
$$

We proceed likewise with the other terms in (29). For

$$
\begin{aligned}
& I_{2}\left(\vec{U}^{\varepsilon}, \vec{U}^{\varepsilon}\right)=\varepsilon \int_{\omega} 4 l^{0} H^{\alpha 3 \beta 3}\left(\zeta_{\alpha}\left(\vec{u}^{\varepsilon}, \vec{\theta}^{\varepsilon}\right) \zeta_{\beta}\left(\vec{u}^{\varepsilon}, \vec{\theta}^{\varepsilon}\right)+\frac{t^{2}}{6} \zeta_{\alpha}\left(\vec{u}^{\varepsilon}, \vec{\theta}^{\varepsilon}\right) n_{\beta}\left(\vec{\varrho}^{\varepsilon}\right)\right. \\
& \left.\quad+\frac{t^{2}}{12} m_{\alpha}\left(\vec{\theta}^{\varepsilon}, \vec{\varrho}^{\varepsilon}\right) m_{\beta}\left(\vec{\theta}^{\varepsilon}, \vec{\varrho}^{\varepsilon}\right)+\frac{t^{4}}{80} n_{\alpha}\left(\vec{\varrho}^{\varepsilon}\right) n_{\beta}\left(\vec{\varrho}^{\varepsilon}\right)\right) \sqrt{a} d \xi^{1} d \xi^{2}+\bar{I}_{2}\left(\vec{U}^{\varepsilon}, \vec{U}^{\varepsilon}\right) \\
& \quad={ }^{0} I_{2}\left(\vec{U}^{\varepsilon}, \vec{U}^{\varepsilon}\right)+\bar{I}_{2}\left(\vec{U}^{\varepsilon}, \vec{U}^{\varepsilon}\right),
\end{aligned}
$$

we can show that $\frac{1}{\varepsilon^{3}} \bar{I}_{2}\left(\vec{U}^{\varepsilon}, \vec{U}^{\varepsilon}\right)=R_{\varepsilon}$, and defining

$$
D_{2, \varepsilon}=\int_{\omega} \frac{l^{3}}{3}{ }^{0} H^{\alpha 3 \lambda 3} m_{\alpha}\left(\vec{\theta}^{\varepsilon}-\vec{\theta}^{0}, \vec{\varrho}^{\varepsilon}-\vec{\varrho}^{0}\right) m_{\beta}\left(\vec{\theta}^{\varepsilon}-\vec{\theta}^{0}, \vec{\varrho}^{\varepsilon}-\vec{\varrho}^{0}\right) d S,
$$

and

$$
D_{2, \varepsilon}^{\prime}=\int_{\omega} \frac{l^{3}}{3}{ }^{0} H^{\alpha 3 \lambda 3} m_{\alpha}\left(\vec{\theta}^{\varepsilon}, \vec{\varrho}^{\varepsilon}\right) m_{\beta}\left(\vec{\theta}^{\varepsilon}, \vec{\varrho}^{\varepsilon}\right) d S
$$

we obtain

$$
D_{2, \varepsilon}=D_{2, \varepsilon}^{\prime}-\int_{\omega} \frac{l^{3}}{3}{ }^{0} H^{\alpha 3 \lambda 3} m_{\alpha}\left(\vec{\theta}^{0}, \vec{\varrho}^{0}\right) m_{\beta}\left(\vec{\theta}^{0}, \vec{\varrho}^{0}\right) d S+R_{\varepsilon},
$$

with

$$
\begin{aligned}
& D_{2, \varepsilon}^{\prime}=\frac{1}{\varepsilon^{3}} 0 I_{2}\left(\vec{U}^{\varepsilon}, \vec{U}^{\varepsilon}\right)-\frac{1}{\varepsilon^{2}} \int_{\omega} 4 l^{0} H^{\alpha 3 \lambda 3} \zeta_{\alpha}\left(\vec{u}^{\varepsilon}, \vec{\theta}^{\varepsilon}\right) \zeta_{\beta}\left(\vec{u}^{\varepsilon}, \vec{\theta}^{\varepsilon}\right) d S \\
&-\int_{\omega} \frac{2 l^{3}}{3}{ }^{0} H^{\alpha 3 \lambda 3} \zeta_{\alpha}\left(\vec{u}^{\varepsilon}, \vec{\theta}^{\varepsilon}\right) n_{\beta}\left(\vec{\varrho}^{\varepsilon}\right) d S-\varepsilon^{2} \int_{\omega} \frac{l^{5}}{20}{ }^{0} H^{\alpha 3 \lambda 3} n_{\alpha}\left(\vec{\varrho}^{\varepsilon}\right) n_{\beta}\left(\vec{\varrho}^{\varepsilon}\right) d S .
\end{aligned}
$$

Next, for

$$
\begin{aligned}
I_{3}\left(\vec{U}^{\varepsilon}, \vec{U}^{\varepsilon}\right) & =\varepsilon \int_{\omega} l{ }^{0} H^{3333}\left(\left(\delta\left(\vec{\theta}^{\varepsilon}\right)\right)^{2}+\frac{t^{2}}{12}\left(p\left(\vec{\varrho}^{\varepsilon}\right)\right)^{2}\right) \sqrt{a} d \xi^{1} d \xi^{2}+\bar{I}_{3}\left(\vec{U}^{\varepsilon}, \vec{U}^{\varepsilon}\right) \\
& ={ }^{0} I_{3}\left(\vec{U}^{\varepsilon}, \vec{U}^{\varepsilon}\right)+\bar{I}_{3}\left(\vec{U}^{\varepsilon}, \vec{U}^{\varepsilon}\right),
\end{aligned}
$$


we have $\frac{1}{\varepsilon^{3}} \bar{I}_{3}\left(\vec{U}^{\varepsilon}, \vec{U}^{\varepsilon}\right)=R_{\varepsilon}$, and defining

$$
D_{3, \varepsilon}=\int_{\omega} \frac{l^{3}}{12}{ }^{0} H^{3333}\left(p\left(\vec{\varrho}^{\varepsilon}\right)-p\left(\vec{\varrho}^{0}\right)\right)^{2} d S
$$

and

$$
D_{3, \varepsilon}^{\prime}=\int_{\omega} \frac{l^{3}}{12}{ }^{0} H^{3333}\left(p\left(\vec{\varrho}^{\varepsilon}\right)\right)^{2} d S
$$

we obtain

$$
D_{3, \varepsilon}=D_{3, \varepsilon}^{\prime}-\int_{\omega} \frac{l^{3}}{12}{ }^{0} H^{3333}\left(p\left(\vec{\varrho}^{0}\right)\right)^{2} d S+R_{\varepsilon},
$$

with

$$
D_{3, \varepsilon}^{\prime}=\frac{1}{\varepsilon^{3}}{ }^{0} I_{3}\left(\vec{U}^{\varepsilon}, \vec{U}^{\varepsilon}\right)-\frac{1}{\varepsilon^{2}} \int_{\omega} l^{0} H^{3333}\left(\delta\left(\vec{\theta}^{\varepsilon}\right)\right)^{2} d S .
$$

Finally, for the last two terms $I_{4}\left(\overrightarrow{U^{\varepsilon}}, \vec{U}^{\varepsilon}\right)=I_{5}\left(\vec{U}^{\varepsilon}, \vec{U}^{\varepsilon}\right)$, denoting

$$
\begin{aligned}
I_{4}\left(\vec{U}^{\varepsilon}, \vec{U}^{\varepsilon}\right)=\varepsilon & \int_{\omega} l^{0} H^{\alpha \beta 33}\left(\gamma_{\alpha \beta}\left(\vec{u}^{\varepsilon}\right) \delta\left(\vec{\theta}^{\varepsilon}\right)+\frac{t^{2}}{12} \hat{\chi}_{\alpha \beta}\left(\vec{u}^{\varepsilon}, \vec{\theta}^{\varepsilon}\right) p\left(\vec{\varrho}^{\varepsilon}\right)\right. \\
& \left.+\frac{t^{2}}{12} k_{\alpha \beta}\left(\vec{\theta}^{\varepsilon}, \vec{\varrho}^{\varepsilon}\right) \delta\left(\vec{\theta}^{\varepsilon}\right)\right) \sqrt{a} d \xi^{1} d \xi^{2}+\bar{I}_{4}\left(\vec{U}^{\varepsilon}, \vec{U}^{\varepsilon}\right) \\
= & { }^{0} I_{4}\left(\vec{U}^{\varepsilon}, \vec{U}^{\varepsilon}\right)+\bar{I}_{4}\left(\vec{U}^{\varepsilon}, \vec{U}^{\varepsilon}\right),
\end{aligned}
$$

we can show that $\frac{1}{\varepsilon^{3}} \bar{I}_{4}\left(\vec{U}^{\varepsilon}, \vec{U}^{\varepsilon}\right)=R_{\varepsilon}$, and with

$$
D_{4, \varepsilon}=\int_{\omega} \frac{l^{3}}{12}{ }^{0} H^{\alpha \beta 33} \hat{\chi}_{\alpha \beta}\left(\vec{u}^{\varepsilon}-\vec{u}^{0}, \vec{\theta}^{\varepsilon}-\vec{\theta}^{0}\right) p\left(\vec{\varrho}^{\varepsilon}-\vec{\varrho}^{0}\right) d S,
$$

and

$$
D_{4, \varepsilon}^{\prime}=\int_{\omega} \frac{l^{3}}{12}{ }^{0} H^{\alpha \beta 33} \hat{\chi}_{\alpha \beta}\left(\vec{u}^{\varepsilon}, \vec{\theta}^{\varepsilon}\right) p\left(\vec{\varrho}^{\varepsilon}\right) d S
$$

we obtain

$$
D_{4, \varepsilon}=D_{4, \varepsilon}^{\prime}-\int_{\omega} \frac{l^{3}}{12}{ }^{0} H^{\alpha \beta 33} \hat{\chi}_{\alpha \beta}\left(\vec{u}^{0}, \vec{\theta}^{0}\right) p\left(\vec{\varrho}^{0}\right) d S+R_{\varepsilon}
$$

with

$$
\begin{array}{r}
D_{4, \varepsilon}^{\prime}=\frac{1}{\varepsilon^{3}} I_{4}\left(\vec{U}^{\varepsilon}, \vec{U}^{\varepsilon}\right)-\frac{1}{\varepsilon^{2}} \int_{\omega} l^{0} H^{\alpha \beta 33} \gamma_{\alpha \beta}\left(\vec{u}^{\varepsilon}\right) \delta\left(\vec{\theta}^{\varepsilon}\right) d S \\
-\int_{\omega} \frac{l^{3}}{12}{ }^{0} H^{\alpha \beta 33} k_{\alpha \beta}\left(\vec{\theta}^{\varepsilon}, \vec{\varrho}^{\varepsilon}\right) \delta\left(\vec{\theta}^{\varepsilon}\right) d S .
\end{array}
$$

Defining now $D_{\varepsilon}=D_{1, \varepsilon}+D_{2, \varepsilon}+D_{3, \varepsilon}+2 D_{4, \varepsilon}, D_{\varepsilon}^{\prime}=D_{1, \varepsilon}^{\prime}+D_{2, \varepsilon}^{\prime}+D_{3, \varepsilon}^{\prime}+2 D_{4, \varepsilon}^{\prime}$ and $\bar{I}=$ $\bar{I}_{1}+\bar{I}_{2}+\bar{I}_{3}+2 \bar{I}_{4}$, and using (40), (42), (44) and (46), we obtain

$$
D_{\varepsilon}^{\prime}=\frac{1}{\varepsilon^{3}} A^{3 D s}\left(\vec{U}^{\varepsilon}, \vec{U}^{\varepsilon}\right)-\frac{\bar{I}}{\varepsilon^{3}}-\tilde{D}_{\varepsilon}
$$


with

$$
\begin{aligned}
\tilde{D}_{\varepsilon} & =\frac{1}{\varepsilon^{2}} \int_{\omega} l^{0} H^{\alpha \beta \lambda \mu} \gamma_{\alpha \beta}\left(\vec{u}^{\varepsilon}\right) \gamma_{\lambda \mu}\left(\vec{u}^{\varepsilon}\right) d S+\int_{\omega} \frac{l^{3}}{6} H^{\alpha \beta \lambda \mu} \gamma_{\alpha \beta}\left(\vec{u}^{\varepsilon}\right) k_{\lambda \mu}\left(\vec{\theta}^{\varepsilon}, \vec{\varrho}^{\varepsilon}\right) d S \\
& +\varepsilon^{2} \int_{\omega} \frac{l^{5}}{80} H^{0} H^{\alpha \beta \lambda \mu} k_{\alpha \beta}\left(\vec{\theta}^{\varepsilon}, \vec{\varrho}^{\varepsilon}\right) k_{\lambda \mu}\left(\vec{\theta}^{\varepsilon}, \vec{\varrho}^{\varepsilon}\right) d S+\frac{1}{\varepsilon^{2}} \int_{\omega} 4 l^{0} H^{\alpha 3 \lambda 3} \zeta_{\alpha}\left(\vec{u}^{\varepsilon}, \vec{\theta}^{\varepsilon}\right) \zeta_{\beta}\left(\vec{u}^{\varepsilon}, \vec{\theta}^{\varepsilon}\right) d S \\
& +\int_{\omega} \frac{2 l^{3}}{3}{ }^{0} H^{\alpha 3 \lambda 3} n_{\alpha}\left(\vec{\varrho}^{\varepsilon}\right) \zeta_{\beta}\left(\vec{u}^{\varepsilon}, \vec{\theta}^{\varepsilon}\right) d S+\varepsilon^{2} \int_{\omega} \frac{l^{5}}{20}{ }^{0} H^{\alpha 3 \lambda 3} n_{\alpha}\left(\vec{\varrho}^{\varepsilon}\right) n_{\beta}\left(\vec{\varrho}^{\varepsilon}\right) d S \\
& +\frac{1}{\varepsilon^{2}} \int_{\omega} l^{0} H^{3333}\left(\delta\left(\vec{\theta}^{\varepsilon}\right)\right)^{2} d S+\frac{2}{\varepsilon^{2}} \int_{\omega} l^{0} H^{\alpha \beta 33} \gamma_{\alpha \beta}\left(\vec{u}^{\varepsilon}\right) \delta\left(\vec{\theta}^{\varepsilon}\right) d S \\
& +2 \int_{\omega} \frac{l^{3}}{12}{ }^{0} H^{\alpha \beta 33} k_{\alpha \beta}\left(\vec{\theta}^{\varepsilon}, \vec{\varrho}^{\varepsilon}\right) \delta\left(\vec{\theta}^{\varepsilon}\right) d S .
\end{aligned}
$$

Using the variational equation (18) and recalling $\varepsilon^{-3} \bar{I}=R_{\varepsilon}$ we get

$$
D_{\varepsilon}^{\prime}=\frac{1}{\varepsilon^{3}} F^{3 D s}\left(\vec{U}^{\varepsilon}\right)-\tilde{D}_{\varepsilon}+R_{\varepsilon},
$$

while we have by using (36) together with (39), (41), (43) and (45)

$$
D_{\varepsilon}=D_{\varepsilon}^{\prime}-A_{\mathrm{b}}^{3 D s}\left(\vec{u}^{0}, \vec{\theta}^{0}, \vec{\varrho}^{0} ; \vec{u}^{0}, \vec{\theta}^{0}, \vec{\varrho}^{0}\right)+R_{\varepsilon}=D_{\varepsilon}^{\prime}-G^{3 D s}\left(\vec{u}^{0}\right)+R_{\varepsilon},
$$

which yields, when substituting (47),

$$
D_{\varepsilon}=\frac{1}{\varepsilon^{3}} F^{3 D s}\left(\vec{U}^{\varepsilon}\right)-G^{3 D s}\left(\vec{u}^{0}\right)-\tilde{D}_{\varepsilon}+R_{\varepsilon}=-\tilde{D}_{\varepsilon}+R_{\varepsilon},
$$

using the weak convergence. We have thus obtained

$$
D_{\varepsilon}+\tilde{D}_{\varepsilon}=R_{\varepsilon} .
$$

Clearly, $D_{\varepsilon} \geq 0$, and to complete the proof we will now show that

$$
\tilde{D}_{\varepsilon} \geq C\left(\frac{1}{\varepsilon^{2}}\|\underline{\underline{\gamma}}\|_{0}^{2}+\frac{1}{\varepsilon^{2}}\|\underline{\zeta}\|_{0}^{2}+\frac{1}{\varepsilon^{2}}\|\delta\|_{0}+\varepsilon^{2}\|\underline{\underline{k}}\|_{0}^{2}+\varepsilon^{2}\|\underline{n}\|_{0}^{2}\right) .
$$

Defining the tensorial components

$$
\left\{\begin{array}{l}
f_{\alpha \beta}=\frac{1}{\varepsilon} \gamma_{\alpha \beta}\left(\vec{u}^{\varepsilon}\right)+\frac{l^{2} \varepsilon}{12} k_{\alpha \beta}\left(\vec{\theta}^{\varepsilon}, \vec{\varrho}^{\varepsilon}\right) \\
f_{\alpha 3}=\frac{1}{\varepsilon} \zeta_{\alpha}\left(\vec{u}^{\varepsilon}, \vec{\theta}^{\varepsilon}\right)+\frac{l^{2} \varepsilon}{12} n_{\alpha}\left(\vec{\varrho}^{\varepsilon}\right) \\
f_{33}=\frac{1}{\varepsilon} \delta\left(\vec{\theta}^{\varepsilon}\right)
\end{array}\right.
$$

we have

$$
\begin{aligned}
\int_{\omega} l^{0} H^{i j k l} f_{i j} f_{k l} d S=\int_{\omega} l^{0} H^{\alpha \beta \lambda \mu} f_{\alpha \beta} f_{\lambda \mu} d S & +2 \int_{\omega} l^{0} H^{\alpha \beta 33} f_{\alpha \beta} f_{33} d S \\
& +4 \int_{\omega} l^{0} H^{\alpha 3 \beta 3} f_{\alpha 3} f_{\beta 3} d S+\int_{\omega} l^{0} H^{3333} f_{33} f_{33} d S,
\end{aligned}
$$

hence,

$$
\begin{aligned}
\tilde{D}_{\varepsilon}= & \int_{\omega} l^{0} H^{i j k l} f_{i j} f_{k l} d S+\varepsilon^{2} \int_{\omega} l^{5}\left(\frac{1}{80}-\frac{1}{144}\right){ }^{0} H^{\alpha \beta \lambda \mu} k_{\alpha \beta}\left(\vec{\theta}^{\varepsilon}, \vec{\varrho}^{\varepsilon}\right) k_{\lambda \mu}\left(\vec{\theta}^{\varepsilon}, \vec{\varrho}^{\varepsilon}\right) d S \\
& +\varepsilon^{2} \int_{\omega} 4 l^{5}\left(\frac{1}{80}-\frac{1}{144}\right){ }^{0} H^{\alpha 3 \lambda 3} n_{\alpha}\left(\vec{\varrho}^{\varepsilon}\right) n_{\beta}\left(\vec{\varrho}^{\varepsilon}\right) d S \\
\geq & C \int_{\omega} l^{0} H^{i j k l} f_{i j} f_{k l} d S,
\end{aligned}
$$


which implies (49). Therefore, due to (48) both $D_{\varepsilon}$ and $\tilde{D}_{\varepsilon}$ tend to zero. Then, with $\tilde{D}_{\varepsilon}$ converging to zero and (38), we directly infer

$$
\frac{1}{\varepsilon}\left\|\vec{u}^{\varepsilon}, \vec{\theta}^{\varepsilon}\right\|_{\mathrm{m}} \stackrel{\varepsilon \rightarrow 0}{\longrightarrow} 0, \quad \varepsilon\left\|\vec{u}^{\varepsilon}, \vec{\theta}^{\varepsilon}, \vec{\varrho}^{\varepsilon}\right\|_{1} \stackrel{\varepsilon \rightarrow 0}{\longrightarrow} 0,
$$

recalling Lemma 1 . Hence, $\left\|\vec{u}^{\varepsilon}, \vec{\theta}^{\varepsilon}\right\|_{\mathrm{m}}$ tends to zero a fortiori, and combined with $D_{\varepsilon}$ converging to zero this finally proves that $\left(\vec{u}^{\varepsilon}, \vec{\theta}^{\varepsilon}, \vec{\varrho}^{\varepsilon}\right)$ converges strongly in $\mathcal{V}_{\mathrm{b}}^{3 D s}$ to $\left(\vec{u}^{0}, \vec{\theta}^{0}, \vec{\varrho}^{0}\right)$, and all the claims of the Proposition are thus established.

Remark 1 The asymptotic behavior is essentially of a penalization type in this case - as for the bending-dominated behavior of classical shell models [8] - since the limit solution lies in the constrained subspace $\mathcal{V}_{0 \mathrm{~b}}^{3 D}$, albeit also with some secondary features of singular perturbation here, since the regularity of $\mathcal{V}_{\mathrm{b}}^{3 D s}$ is lower than that of the original space $\mathcal{V}^{3 D s}$, except for the first-order term $\vec{v}$ in the polynomial expression, recall (27).

Remark 2 The displacement of any point within the shell body being given by

$$
\left.\vec{U}^{\varepsilon}=\vec{u}^{\varepsilon}\left(\xi^{1}, \xi^{2}\right)+\xi^{3} \vec{\theta}^{\varepsilon}\left(\xi^{1}, \xi^{2}\right)+\left(\xi^{3}\right)^{2} \vec{\varrho}^{\varepsilon}\left(\xi^{1}, \xi^{2}\right), \quad \xi^{3} \in \varepsilon \times\right]-\frac{l\left(\xi^{1}, \xi^{2}\right)}{2},+\frac{l\left(\xi^{1}, \xi^{2}\right)}{2}[,
$$

the additional convergence results (50) directly imply uniform convergence properties of $\vec{U}^{\varepsilon}$ to $\vec{u}^{0}$ in $H^{1}(\mathcal{S})$, e.g., when taking $\xi^{3} /(\varepsilon l)$ as a parameter, such as for the the top and bottom surfaces of the shell.

\subsection{Inhibited pure bending}

We now assume

$$
\mathcal{V}_{0}^{3 D s} \cap\left\{(\vec{v}, \vec{\eta}, \overrightarrow{0}) \in \mathcal{V}^{3 D s}\right\}=\{(\overrightarrow{0}, \overrightarrow{0}, \overrightarrow{0})\},
$$

in which case $\|\vec{v}, \vec{\eta}\|_{\mathrm{m}}$ gives a norm, and we accordingly introduce $\mathcal{V}_{\mathrm{m}}^{3 D s}$ as the completion of $\mathcal{V}^{\natural}=\left\{(\vec{v}, \vec{\eta})\right.$ such that $\left.(\vec{v}, \vec{\eta}, \overrightarrow{0}) \in \mathcal{V}^{3 D s}\right\}$ with respect to this norm. The convergence of the asymptotic behavior will then be obtained in this space for the scaling corresponding to $\rho=1$, and the candidate limit problem reads:

Find $\left(\vec{u}^{\mathrm{m}}, \vec{\theta}^{\mathrm{m}}\right) \in \mathcal{V}_{\mathrm{m}}^{3 D s}$ such that

$$
A_{\mathrm{m}}^{3 D s}\left(\vec{u}^{\mathrm{m}}, \vec{\theta}^{\mathrm{m}} ; \vec{v}, \vec{\eta}\right)=G^{3 D s}(\vec{v}), \quad \forall(\vec{v}, \vec{\eta}) \in \mathcal{V}_{\mathrm{m}}^{3 D s} .
$$

We point out that, in order to obtain a well-posed limit problem, we need to enforce that $G^{3 D s} \in\left(\mathcal{V}_{\mathrm{m}}^{3 D s}\right)^{\prime}$, namely, that

$$
\left|\int_{\omega} l \vec{G}_{0} \cdot \vec{v} d S\right| \leq C\|\vec{v}, \vec{\eta}\|_{\mathrm{m}}, \quad \forall(\vec{v}, \vec{\eta}) \in \mathcal{V}_{\mathrm{m}}^{3 D s},
$$

which we call the condition of admissible loading for membrane-dominated behavior, see $[8,21]$ and references therein for discussions on this condition. Provided this condition is satisfied, the variational problem (52) is clearly well-posed, by construction.

We start by proving weak convergence, in a more detailed form than in $[9,8]$.

Lemma 3 Assuming that $G^{3 D s} \in\left(\mathcal{V}_{\mathrm{m}}^{3 D s}\right)^{\prime}$, we have that $\left(\vec{u}^{\varepsilon}, \vec{\theta}^{\varepsilon}\right)$ converges weakly in $\mathcal{V}_{\mathrm{m}}^{3 D s}$, as $\varepsilon$ goes to 0 , to $\left(\vec{u}^{\mathrm{m}}, \vec{\theta}^{\mathrm{m}}\right)$ solution of $(52)$. Moreover, $\varepsilon^{2} \vec{\varrho}^{\varepsilon}$ converges weakly in $H^{1}(\mathcal{S})$ to 0 . 
Proof We divide the proof into two steps.

i) Extraction of weakly-converging sequence and preliminary identifications. We can establish the following uniform bound on the sequence of solutions $[9,8]$

$$
\left\|\vec{u}^{\varepsilon}, \vec{\theta}^{\varepsilon}\right\|_{\mathrm{m}}+\varepsilon\left\|\vec{u}^{\varepsilon}, \vec{\theta}^{\varepsilon}, \vec{\varrho}^{\varepsilon}\right\|_{\mathrm{b}}+\varepsilon^{2}\left\|\vec{u}^{\varepsilon}, \vec{\theta}^{\varepsilon}, \vec{\varrho}^{\varepsilon}\right\|_{1} \leq C .
$$

Hence, we can extract a subsequence - also denoted by $\left(\vec{u}^{\varepsilon}, \vec{\theta}^{\varepsilon}, \vec{\varrho}^{\varepsilon}\right)$ for convenience - such that we have the weak convergence of $\left(\vec{u}^{\varepsilon}, \vec{\theta}^{\varepsilon}\right)$ to some $\left(\vec{u}^{w}, \vec{\theta}^{w}\right)$ in $\mathcal{V}_{\mathrm{m}}^{3 D s}$, together with the weak convergence of $\varepsilon^{2} \vec{\varrho}^{\varepsilon}$ to some $\vec{\varrho}^{w}$ in $H^{1}(\mathcal{S})$. We will now show that

$$
\vec{\varrho}^{w}=0 .
$$

Recalling the second equivalence of norms in Lemma 1 and the expressions of $m_{\alpha}$ and $p$ in (15), the boundedness of $\varepsilon\left\|\vec{u}^{\varepsilon}, \vec{\theta}^{\varepsilon}, \vec{\varrho}^{\varepsilon}\right\|_{\mathrm{b}}$ implies that $\varepsilon\left(2 \varrho_{\alpha}^{\varepsilon}+\theta_{3, \alpha}^{\varepsilon}\right)$ and $\varepsilon \varrho_{3}^{\varepsilon}$ are both bounded in $L^{2}(\mathcal{S})$. Therefore, $\varepsilon^{2}\left(2 \varrho_{\alpha}^{\varepsilon}+\theta_{3, \alpha}^{\varepsilon}\right)$ and $\varepsilon^{2} \varrho_{3}^{\varepsilon}$ tend to zero in $L^{2}(\mathcal{S})$, which already shows that $\rho_{3}^{w}=0$. Moreover, since $\varepsilon^{2} \rho_{\alpha}$ tends to $\rho_{\alpha}^{w}$ in $L^{2}(\mathcal{S})$, we infer that $\varepsilon^{2} \theta_{3, \alpha}^{\varepsilon}$ tends to $-2 \rho_{\alpha}^{w}$ in $L^{2}(\mathcal{S})$. On the other hand, the boundedness of $\left\|\vec{u}^{\varepsilon}, \vec{\theta}^{\varepsilon}\right\|_{\mathrm{m}}$ implies that $\theta_{3}^{\varepsilon}$ is bounded in $L^{2}(\mathcal{S})$. Hence, $\varepsilon^{2} \theta_{3}^{\varepsilon}$ tends to zero in $L^{2}(\mathcal{S})$, thus also in the distribution sense. As a consequence, $\varepsilon^{2} \theta_{3, \alpha}^{\varepsilon}$ also tends to zero in the distribution sense, which shows that $\rho_{\alpha}^{w}=0$, hence (55) is proven.

ii) Identification of weak limit $\left(\vec{u}^{w}, \vec{\theta}^{w}\right)$. We will again use the decomposition (29), and similar notation as in the proof of Proposition 2 for

$$
I_{i}(\vec{U}, \vec{V})={ }^{0} I_{i}(\vec{U}, \vec{V})+\bar{I}_{i}(\vec{U}, \vec{V}), \quad i=1, \ldots, 5,
$$

where of course all the mixed terms present in the above-defined expressions of ${ }^{0} I_{i}(\vec{U}, \vec{U})$ are symmetrized in ${ }^{0} I_{i}(\vec{U}, \vec{V})$. Starting with the first term, we have

$$
\begin{gathered}
{ }^{0} I_{1}\left(\vec{U}^{\varepsilon}, \vec{V}\right)=\varepsilon \int_{\omega} l^{0} H^{\alpha \beta \lambda \mu}\left(\gamma_{\alpha \beta}\left(\vec{u}^{\varepsilon}\right) \gamma_{\lambda \mu}(\vec{v})+\frac{t^{2}}{12} \gamma_{\alpha \beta}\left(\vec{u}^{\varepsilon}\right) k_{\lambda \mu}(\vec{\eta}, \vec{\varsigma})\right. \\
+\frac{t^{2}}{12} \gamma_{\alpha \beta}(\vec{v}) k_{\lambda \mu}\left(\vec{\theta}^{\varepsilon}, \vec{\varrho}^{\varepsilon}\right)+\frac{t^{2}}{12} \hat{\chi}_{\alpha \beta}\left(\vec{u}^{\varepsilon}, \vec{\theta}^{\varepsilon}\right) \hat{\chi} \lambda \mu(\vec{v}, \vec{\eta}) \\
\left.+\frac{t^{4}}{80} k_{\alpha \beta}\left(\vec{\theta}^{\varepsilon}, \vec{\varrho}^{\varepsilon}\right) k_{\lambda \mu}(\vec{\eta}, \vec{\varsigma})\right) d S,
\end{gathered}
$$

and we can easily show for the remainder that $\varepsilon^{-1} \bar{I}_{1}$ tends to zero based on (54). Concerning $\varepsilon^{-10} I_{1}$, the weak convergence of $\left(\vec{u}^{\varepsilon}, \vec{\theta}^{\varepsilon}\right)$ implies that the first term under the integral tends to

$$
\int_{\omega} l^{0} H^{\alpha \beta \lambda \mu} \gamma_{\alpha \beta}\left(\vec{u}^{w}\right) \gamma_{\lambda \mu}(\vec{v}) d S
$$

and the uniform bound (54) directly implies that all other terms tend to zero, except for the third one which requires further analysis. We have

$$
\varepsilon^{2} k_{\lambda \mu}\left(\vec{\theta}^{\varepsilon}, \vec{\varrho}^{\varepsilon}\right)=\varepsilon^{2}\left(\frac{1}{2}\left(\varrho_{\lambda \mid \mu}^{\varepsilon}+\varrho_{\mu \mid \lambda}^{\varepsilon}\right)-b_{\lambda \mu} \varrho_{3}^{\varepsilon}-\frac{1}{2}\left(b_{\lambda}^{\alpha} \theta_{\alpha \mid \mu}^{\varepsilon}+b_{\mu}^{\alpha} \theta_{\alpha \mid \lambda}^{\varepsilon}\right)+c_{\lambda \mu} \theta_{3}^{\varepsilon}\right)
$$

and the weak convergence of $\varepsilon^{2} \vec{\varrho}^{\varepsilon}$ to zero in $H^{1}(\mathcal{S})$ implies that the terms with $\vec{\varrho}^{\varepsilon}$ tend to zero weakly in $L^{2}(\mathcal{S})$. Then from the boundedness of $\varepsilon\left\|\vec{u}^{\varepsilon}, \vec{\theta}^{\varepsilon}, \vec{\varrho}^{\varepsilon}\right\|_{\mathrm{b}}$ in (54) and recalling (27) we infer that the terms with $\vec{\theta}^{\varepsilon}$ tend to zero strongly in $L^{2}(\mathcal{S})$. This shows that all terms but the first one vanish in $\varepsilon^{-10} I_{1}$ in the limit, hence

$$
\varepsilon^{-1} I_{1}\left(\vec{U}^{\varepsilon}, \vec{V}\right) \stackrel{\varepsilon \rightarrow 0}{\longrightarrow} \int_{\omega} l^{0} H^{\alpha \beta \lambda \mu} \gamma_{\alpha \beta}\left(\vec{u}^{w}\right) \gamma_{\lambda \mu}(\vec{v}) d S .
$$


All the other terms in the decomposition (29) can be handled with very similar arguments, which leads to

$$
\begin{gathered}
\varepsilon^{-1} I_{2}\left(\vec{U}^{\varepsilon}, \vec{V}\right) \stackrel{\varepsilon \rightarrow 0}{\longrightarrow} \int_{\omega} 4 l^{0} H^{\alpha 3 \beta 3} \zeta_{\alpha}\left(\vec{u}^{w}, \vec{\theta}^{w}\right) \zeta_{\beta}(\vec{v}, \vec{\eta}) d S, \\
\varepsilon^{-1} I_{3}\left(\vec{U}^{\varepsilon}, \vec{V}\right) \stackrel{\varepsilon \rightarrow 0}{\longrightarrow} \int_{\omega} l^{0} H^{3333} \delta\left(\vec{\theta}^{w}\right) \delta(\vec{\eta}) d S, \\
\varepsilon^{-1} I_{4}\left(\vec{U}^{\varepsilon}, \vec{V}\right) \stackrel{\varepsilon \rightarrow 0}{\longrightarrow} \int_{\omega} l^{0} H^{\alpha \beta 33} \gamma_{\alpha \beta}\left(\vec{u}^{w}\right) \delta(\vec{\eta}) d S, \\
\varepsilon^{-1} I_{5}\left(\vec{U}^{\varepsilon}, \vec{V}\right) \stackrel{\varepsilon \rightarrow 0}{\longrightarrow} \int_{\omega} l^{0} H^{\alpha \beta 33} \gamma_{\alpha \beta}(\vec{v}) \delta\left(\vec{\theta}^{w}\right) d S .
\end{gathered}
$$

Therefore, in the variational formulation (18) we have

$$
\varepsilon^{-1} A^{3 D s}\left(\vec{u}^{\varepsilon}, \vec{\theta}^{\varepsilon}, \vec{\varrho}^{\varepsilon} ; \vec{v}, \vec{\eta}, \vec{\varsigma}\right) \stackrel{\varepsilon \rightarrow 0}{\longrightarrow} A_{\mathrm{m}}^{3 D s}\left(\vec{u}^{w}, \vec{\theta}^{w} ; \vec{v}, \vec{\eta}\right) .
$$

Moreover, it is straightforward to show that

$$
\varepsilon^{-1} F^{3 D s}(\vec{v}, \vec{\eta}, \vec{\varsigma}) \stackrel{\varepsilon \rightarrow 0}{\longrightarrow} G^{3 D s}(\vec{v}) .
$$

Finally, taking the limit of (18) multiplied by $\varepsilon^{-1}$, we see that $\left(\vec{u}^{w}, \vec{\theta}^{w}\right)$ satisfies

$$
A_{\mathrm{m}}^{3 D s}\left(\vec{u}^{w}, \vec{\theta}^{w} ; \vec{v}, \vec{\eta}\right)=G^{3 D s}(\vec{v}),
$$

for any $(\vec{v}, \vec{\eta}) \in \mathcal{V}^{\natural}$, hence also for any $(\vec{v}, \vec{\eta}) \in \mathcal{V}_{\mathrm{m}}^{3 D s}$ since $\mathcal{V}^{\natural}$ is dense in $\mathcal{V}_{\mathrm{m}}^{3 D s}$ by construction of the latter space. This shows that $\left(\vec{u}^{w}, \vec{\theta}^{w}\right)=\left(\vec{u}^{\mathrm{m}}, \vec{\theta}^{\mathrm{m}}\right)$ for the weak convergence of the extracted subsequence, hence this convergence holds for the whole original sequence since the limit is hereby uniquely characterized.

We are now in a position to prove the final result of this paper, namely, that the convergence is in fact strong in this case also.

Proposition 3 Assuming that $G^{3 D s} \in\left(\mathcal{V}_{\mathrm{m}}^{3 D s}\right)^{\prime}$, we have that $\left(\vec{u}^{\varepsilon}, \vec{\theta}^{\varepsilon}\right)$ converges strongly in $\mathcal{V}_{\mathrm{m}}^{3 D s}$, as $\varepsilon$ goes to 0 , to $\left(\vec{u}^{\mathrm{m}}, \vec{\theta}^{\mathrm{m}}\right)$ solution of $(52)$. Furthermore, $\varepsilon\left\|\vec{u}^{\varepsilon}, \vec{\theta}^{\varepsilon}, \vec{\varrho}^{\varepsilon}\right\|_{\mathrm{b}}$ and $\varepsilon^{2}\left\|\vec{u}^{\varepsilon}, \vec{\theta}^{\varepsilon}, \vec{\varrho}^{\varepsilon}\right\|_{1}$ both tend to zero.

Proof We again use the decomposition (29) and the splitting (56), both defined as in the proof of Proposition 2. Starting with $I_{1}\left(\vec{U}^{\varepsilon}, \vec{U}^{\varepsilon}\right)$, based on (54) we can now easily see that $\varepsilon^{-1} \bar{I}_{1}\left(\vec{U}^{\varepsilon}, \vec{U}^{\varepsilon}\right)=R_{\varepsilon}$. We then define the following new quantities (different from those introduced in the proof of Proposition 2 with the same notation), where the key point of this proof lies in an appropriate combination of strain tensors at various orders, viz.

$$
D_{1, \varepsilon}=\int_{\omega} l^{0} H^{\alpha \beta \lambda \mu}\left(\gamma_{\alpha \beta}\left(\vec{u}^{\varepsilon}-\vec{u}^{\mathrm{m}}\right)+\frac{t^{2}}{12} k_{\alpha \beta}\left(\vec{\theta}^{\varepsilon}, \vec{\varrho}^{\varepsilon}\right)\right)\left(\gamma_{\lambda \mu}\left(\vec{u}^{\varepsilon}-\vec{u}^{\mathrm{m}}\right)+\frac{t^{2}}{12} k_{\lambda \mu}\left(\vec{\theta}^{\varepsilon}, \vec{\varrho}^{\varepsilon}\right)\right) d S,
$$

and

$$
D_{1, \varepsilon}^{\prime}=\int_{\omega} l^{0} H^{\alpha \beta \lambda \mu}\left(\gamma_{\alpha \beta}\left(\vec{u}^{\varepsilon}\right)+\frac{t^{2}}{12} k_{\alpha \beta}\left(\vec{\theta}^{\varepsilon}, \vec{\varrho}^{\varepsilon}\right)\right)\left(\gamma_{\lambda \mu}\left(\vec{u}^{\varepsilon}\right)+\frac{t^{2}}{12} k_{\lambda \mu}\left(\vec{\theta}^{\varepsilon}, \vec{\varrho}^{\varepsilon}\right)\right) d S,
$$

and we have

$D_{1, \varepsilon}=D_{1, \varepsilon}^{\prime}+\int_{\omega} l^{0} H^{\alpha \beta \lambda \mu} \gamma_{\alpha \beta}\left(\vec{u}^{\mathrm{m}}\right) \gamma_{\lambda \mu}\left(\vec{u}^{\mathrm{m}}\right) d S-\int_{\omega} 2 l^{0} H^{\alpha \beta \lambda \mu}\left(\gamma_{\alpha \beta}\left(\vec{u}^{\varepsilon}\right)+\frac{t^{2}}{12} k_{\alpha \beta}\left(\vec{\theta}^{\varepsilon}, \vec{\varrho}^{\varepsilon}\right)\right) \gamma_{\lambda \mu}\left(\vec{u}^{\mathrm{m}}\right) d S$. 
Using the weak convergence properties of Lemma 3 and (54), we infer that

$$
\int_{\omega} 2 l^{0} H^{\alpha \beta \lambda \mu}\left(\gamma_{\alpha \beta}\left(\vec{u}^{\varepsilon}\right)+\frac{t^{2}}{12} k_{\alpha \beta}\left(\vec{\theta}^{\varepsilon}, \vec{\varrho}^{\varepsilon}\right)\right) \gamma_{\lambda \mu}\left(\vec{u}^{\mathrm{m}}\right) d S \stackrel{\varepsilon \rightarrow 0}{\longrightarrow} \int_{\omega} 2 l^{0} H^{\alpha \beta \lambda \mu} \gamma_{\alpha \beta}\left(\vec{u}^{\mathrm{m}}\right) \gamma_{\lambda \mu}\left(\vec{u}^{\mathrm{m}}\right) d S,
$$

hence,

$$
D_{1, \varepsilon}=D_{1, \varepsilon}^{\prime}-\int_{\omega} l^{0} H^{\alpha \beta \lambda \mu} \gamma_{\alpha \beta}\left(\vec{u}^{\mathrm{m}}\right) \gamma_{\lambda \mu}\left(\vec{u}^{\mathrm{m}}\right) d S+R_{\varepsilon} .
$$

and $D_{1, \varepsilon}^{\prime}$ is related to ${ }^{0} I_{1}\left(\vec{U}^{\varepsilon}, \vec{U}^{\varepsilon}\right)$ by

$$
D_{1, \varepsilon}^{\prime}=\frac{1_{0}}{\varepsilon} I_{1}\left(\vec{U}^{\varepsilon}, \vec{U}^{\varepsilon}\right)-\int_{\omega} l^{0} H^{\alpha \beta \lambda \mu}\left(\frac{t^{2}}{12} \hat{\chi}_{\alpha \beta}\left(\vec{u}^{\varepsilon}, \vec{\theta}^{\varepsilon}\right) \hat{\chi}_{\lambda \mu}\left(\vec{u}^{\varepsilon}, \vec{\theta}^{\varepsilon}\right)+\frac{t^{4}}{180} k_{\alpha \beta}\left(\vec{\theta}^{\varepsilon}, \vec{\varrho}^{\varepsilon}\right) k_{\lambda \mu}\left(\vec{\theta}^{\varepsilon}, \vec{\varrho}^{\varepsilon}\right)\right) d S .
$$

Proceeding similarly for the other terms in the decomposition (29), we have

$$
\varepsilon^{-1} \bar{I}_{i}\left(\vec{U}^{\varepsilon}, \vec{U}^{\varepsilon}\right)=R_{\varepsilon}, \quad i=1, \ldots, 5 .
$$

Also, defining

$$
\begin{gathered}
D_{2, \varepsilon}=\int_{\omega} 4 l^{0} H^{\alpha 3 \beta 3}\left(\zeta_{\alpha}\left(\vec{u}^{\varepsilon}-\vec{u}^{\mathrm{m}}, \vec{\theta}^{\varepsilon}-\vec{\theta}^{\mathrm{m}}\right)+\frac{t^{2}}{12} n_{\alpha}\left(\vec{\varrho}^{\varepsilon}\right)\right)\left(\zeta_{\beta}\left(\vec{u}^{\varepsilon}-\vec{u}^{\mathrm{m}}, \vec{\theta}^{\varepsilon}-\vec{\theta}^{\mathrm{m}}\right)+\frac{t^{2}}{12} n_{\beta}\left(\vec{\varrho}^{\varepsilon}\right)\right) d S, \\
D_{2, \varepsilon}^{\prime}=\int_{\omega} 4 l^{0} H^{\alpha 3 \beta 3}\left(\zeta_{\alpha}\left(\vec{u}^{\varepsilon}, \vec{\theta}^{\varepsilon}\right)+\frac{t^{2}}{12} n_{\alpha}\left(\vec{\varrho}^{\varepsilon}\right)\right)\left(\zeta_{\beta}\left(\vec{u}^{\varepsilon}, \vec{\theta}^{\varepsilon}\right)+\frac{t^{2}}{12} n_{\beta}\left(\vec{\varrho}^{\varepsilon}\right)\right) d S
\end{gathered}
$$

we obtain

$$
\begin{gathered}
D_{2, \varepsilon}=D_{2, \varepsilon}^{\prime}-\int_{\omega} 4 l^{0} H^{\alpha 3 \beta 3} \zeta_{\alpha}\left(\vec{u}^{\mathrm{m}}, \vec{\theta}^{\mathrm{m}}\right) \zeta_{\beta}\left(\vec{u}^{\mathrm{m}}, \vec{\theta}^{\mathrm{m}}\right) d S+R_{\varepsilon}, \\
D_{2, \varepsilon}^{\prime}=\frac{1}{\varepsilon} I_{2}\left(\vec{U}^{\varepsilon}, \vec{U}^{\varepsilon}\right)-\int_{\omega} l^{0} H^{\alpha 3 \beta 3}\left(\frac{t^{2}}{12} m_{\alpha}\left(\vec{\theta}^{\varepsilon}, \vec{\varrho}^{\varepsilon}\right) m_{\beta}\left(\vec{\theta}^{\varepsilon}, \vec{\varrho}^{\varepsilon}\right)+\frac{t^{4}}{180} n_{\alpha}\left(\vec{\varrho}^{\varepsilon}\right) n_{\beta}\left(\vec{\varrho}^{\varepsilon}\right)\right) d S .
\end{gathered}
$$

Likewise, for

$$
D_{3, \varepsilon}=\int_{\omega} l^{0} H^{3333}\left(\delta\left(\vec{\theta}^{\varepsilon}-\vec{\theta}^{\mathrm{m}}\right)\right)^{2} d S, \quad D_{3, \varepsilon}^{\prime}=\int_{\omega} l^{0} H^{3333}\left(\delta\left(\vec{\theta}^{\varepsilon}\right)\right)^{2} d S
$$

we have

$$
\begin{gathered}
D_{3, \varepsilon}=D_{3, \varepsilon}^{\prime}-\int_{\omega} l^{0} H^{3333}\left(\delta\left(\vec{\theta}^{\mathrm{m}}\right)\right)^{2} d S+R_{\varepsilon}, \\
D_{3, \varepsilon}^{\prime}=\frac{1^{0}}{\varepsilon} I_{3}\left(\vec{U}^{\varepsilon}, \vec{U}^{\varepsilon}\right)-\int_{\omega} l^{0} H^{3333} \frac{t^{2}}{12}\left(p\left(\vec{\varrho}^{\varepsilon}\right)\right)^{2} d S .
\end{gathered}
$$

Finally, for

$$
\begin{gathered}
D_{4, \varepsilon}=\int_{\omega} l^{0} H^{\alpha \beta 33}\left(\gamma_{\alpha \beta}\left(\vec{u}^{\varepsilon}-\vec{u}^{\mathrm{m}}\right)+\frac{t^{2}}{12} k_{\alpha \beta}\left(\vec{\theta}^{\varepsilon}, \vec{\varrho}^{\varepsilon}\right)\right) \delta\left(\vec{\theta}^{\varepsilon}-\vec{\theta}^{\mathrm{m}}\right) d S, \\
D_{4, \varepsilon}^{\prime}=\int_{\omega} l^{0} H^{\alpha \beta 33}\left(\gamma_{\alpha \beta}\left(\vec{u}^{\varepsilon}\right)+\frac{t^{2}}{12} k_{\alpha \beta}\left(\vec{\theta}^{\varepsilon}, \vec{\varrho}^{\varepsilon}\right)\right) \delta\left(\vec{\theta}^{\varepsilon}\right) d S,
\end{gathered}
$$

we infer

$$
\begin{gathered}
D_{4, \varepsilon}=D_{4, \varepsilon}^{\prime}-\int_{\omega} l^{0} H^{\alpha \beta 33} \gamma_{\alpha \beta}\left(\vec{u}^{\mathrm{m}}\right) \delta\left(\vec{\theta}^{\mathrm{m}}\right) d S+R_{\varepsilon}, \\
D_{4, \varepsilon}^{\prime}=\frac{1^{0}}{\varepsilon} I_{4}\left(\vec{U}^{\varepsilon}, \vec{U}^{\varepsilon}\right)-\int_{\omega} l^{0} H^{\alpha \beta 33} \frac{t^{2}}{12} \hat{\chi}_{\alpha \beta}\left(\vec{u}^{\varepsilon}, \vec{\theta}^{\varepsilon}\right) p\left(\vec{\varrho}^{\varepsilon}\right) d S .
\end{gathered}
$$


We again define $D_{\varepsilon}=D_{1, \varepsilon}+D_{2, \varepsilon}+D_{3, \varepsilon}+2 D_{4, \varepsilon}, D_{\varepsilon}^{\prime}=D_{1, \varepsilon}^{\prime}+D_{2, \varepsilon}^{\prime}+D_{3, \varepsilon}^{\prime}+2 D_{4, \varepsilon}^{\prime}$ and $\bar{I}=$ $\bar{I}_{1}+\bar{I}_{2}+\bar{I}_{3}+2 \bar{I}_{4}$, and we recall that $\varepsilon^{-1} \bar{I}=R_{\varepsilon}$. Gathering now (63), (65), (67) and (69), we get

$$
D_{\varepsilon}^{\prime}=\frac{1}{\varepsilon} A^{3 D s}\left(\vec{U}^{\varepsilon}, \vec{U}^{\varepsilon}\right)-\frac{1}{\varepsilon} \bar{I}\left(\vec{U}^{\varepsilon}, \vec{U}^{\varepsilon}\right)-\tilde{D}_{\varepsilon},
$$

for

$$
\begin{aligned}
\tilde{D}_{\varepsilon} & =\int_{\omega} l^{0} H^{\alpha \beta \lambda \mu}\left(\frac{t^{2}}{12} \hat{\chi}_{\alpha \beta}\left(\vec{u}^{\varepsilon}, \vec{\theta}^{\varepsilon}\right) \hat{\chi}_{\lambda \mu}\left(\vec{u}^{\varepsilon}, \vec{\theta}^{\varepsilon}\right)+\frac{t^{4}}{180} k_{\alpha \beta}\left(\vec{\theta}^{\varepsilon}, \vec{\varrho}^{\varepsilon}\right) k_{\lambda \mu}\left(\vec{\theta}^{\varepsilon}, \vec{\varrho}^{\varepsilon}\right)\right) d S \\
& +\int_{\omega} l^{0} H^{\alpha 3 \beta 3}\left(\frac{t^{2}}{12} m_{\alpha}\left(\vec{\theta}^{\varepsilon}, \vec{\varrho}^{\varepsilon}\right) m_{\beta}\left(\vec{\theta}^{\varepsilon}, \vec{\varrho}^{\varepsilon}\right)+\frac{t^{4}}{180} n_{\alpha}\left(\vec{\varrho}^{\varepsilon}\right) n_{\beta}\left(\vec{\varrho}^{\varepsilon}\right)\right) d S \\
& +\int_{\omega} l^{0} H^{3333} \frac{t^{2}}{12}\left(p\left(\vec{\varrho}^{\varepsilon}\right)\right)^{2} d S+2 \int_{\omega} l^{0} H^{\alpha \beta 33} \frac{t^{2}}{12} \hat{\chi}_{\alpha \beta}\left(\vec{u}^{\varepsilon}, \vec{\theta}^{\varepsilon}\right) p\left(\vec{\varrho}^{\varepsilon}\right) d S .
\end{aligned}
$$

hence,

$$
D_{\varepsilon}^{\prime}=\frac{1}{\varepsilon} F^{3 D s}\left(\vec{U}^{\varepsilon}\right)-\tilde{D}_{\varepsilon}+R_{\varepsilon}
$$

using the variational equation (18). On the other hand, using (52) together with (62), (64), (66) and (68) we have

$$
D_{\varepsilon}=D_{\varepsilon}^{\prime}-A_{\mathrm{m}}^{3 D s}\left(\vec{u}^{\mathrm{m}}, \vec{\theta}^{\mathrm{m}} ; \vec{u}^{\mathrm{m}}, \vec{\theta}^{\mathrm{m}}\right)+R_{\varepsilon}=D_{\varepsilon}^{\prime}-G^{3 D s}\left(\vec{u}^{\mathrm{m}}\right)+R_{\varepsilon} .
$$

This gives when substituting (70)

$$
D_{\varepsilon}=\frac{1}{\varepsilon} F^{3 D s}\left(\vec{U}^{\varepsilon}\right)-\tilde{D}_{\varepsilon}-G^{3 D s}\left(\vec{u}^{\mathrm{m}}\right)+R_{\varepsilon}=-\tilde{D}_{\varepsilon}+R_{\varepsilon},
$$

invoking the weak convergence. We have thus found

$$
D_{\varepsilon}+\tilde{D}_{\varepsilon}=R_{\varepsilon}
$$

with $D_{\varepsilon} \geq 0$ by construction, and it is straightforward to see that

$$
\tilde{D}_{\varepsilon} \geq C\left(\varepsilon^{2}\|\underline{\underline{\hat{\chi}}}\|_{0}^{2}+\varepsilon^{2}\|\underline{m}\|_{0}^{2}+\varepsilon^{2}\|p\|_{0}^{2}+\varepsilon^{4}\|\underline{\underline{k}}\|_{0}^{2}+\varepsilon^{4}\|\underline{n}\|_{0}^{2}\right) .
$$

Therefore, we infer that $D_{\varepsilon}$ and $\tilde{D}_{\varepsilon}$ both tend to zero. Then, (72) implies that all the terms with coefficient $t^{2}$ appearing in the definitions of $D_{1, \varepsilon}, D_{2, \varepsilon}$ and $D_{4, \varepsilon}$ tend to zero, hence $D_{\varepsilon}$ tending to zero leads to $\left\|\vec{u}^{\varepsilon}-\vec{u}^{\mathrm{m}}, \vec{\theta}^{\varepsilon}-\vec{\theta}^{\mathrm{m}}\right\|_{\mathrm{m}} \stackrel{\varepsilon \rightarrow 0}{\longrightarrow} 0$. Finally, (72) also shows the additional convergence properties

$$
\varepsilon\left\|\vec{u}^{\varepsilon}, \vec{\theta}^{\varepsilon}, \vec{\varrho}^{\varepsilon}\right\|_{\mathrm{b}} \stackrel{\varepsilon \rightarrow 0}{\longrightarrow} 0, \quad \varepsilon^{2}\left\|\vec{u}^{\varepsilon}, \vec{\theta}^{\varepsilon}, \vec{\varrho}^{\varepsilon}\right\|_{1} \stackrel{\varepsilon \rightarrow 0}{\longrightarrow} 0,
$$

recalling the equivalences of norms stated in Lemma 1.

Remark 3 The asymptotic behavior is entirely of singular perturbation type in this case [17], albeit with perturbation terms arising at several orders and with combinations thereof, which requires some rather subtle handling of these combinations in the convergence proofs.

Remark 4 In this case also, we have convergence of the displacements within the shell body - as expressed in (51) - to the limit solution $\vec{u}^{\mathrm{m}}$, uniformly with respect to the parameter given by $\xi^{3} /(\varepsilon l)$, albeit here in the norm provided by $\|\underline{\gamma}\|_{0}$. This is a straightforward consequence of the additional convergence properties given in $(7 \overline{\overline{3}})$, indeed. 


\section{Concluding remarks}

We have revisited the convergence properties of the 3D-shell model proposed in [9], and established strong convergence results for the two main types of asymptotic regimes, namely, bending- and membrane-dominated behavior. This is an important result, as it completely substantiates the asymptotic consistency of the 3D-shell model with 3D elasticity.

As mentioned in the introduction, the finite element discretization of the 3D-shell model is quite straightforward - even more so than for general shell elements due to the possibility of directly employing a general 3D constitutive law in the 3D-shell finite element formulation - and these elements are already available in some widely-used finite element software ${ }^{1}$, indeed. Of course, special care must be exerted to avoid the very serious numerical pathologies associated with shell model discretizations - numerical locking phenomena, in particular - albeit the effective techniques already designed for general shell elements can be rather directly extended to $3 \mathrm{D}$-shells $[8,15,10]$.

\section{References}

[1] S. Ahmad, B.M. Irons, and O.C. Zienkiewicz. Analysis of thick and thin shell structures by curved finite elements. Internat. J. Numer. Methods Engrg., 2:419-451, 1970.

[2] F. Auricchio, C. Lovadina, and A.L. Madureira. An asymptotically optimal model for isotropic heterogeneous linearly elastic plates. ESAIM: M2AN, 38:877-897, 2004.

[3] K.J. Bathe. Finite Element Procedures. Prentice Hall, Englewood Cliffs, 1996.

[4] M. Bernadou. Finite Element Methods for Thin Shell Problems. John Wiley \& Sons, New York, 1996.

[5] M. Bischoff and E. Ramm. On the physical significance of higher order kinematic and static variables in a three-dimensional shell formulation. Internat. J. Solids Structures, 37:6933-6960, 2000.

[6] D. Chapelle and K.J. Bathe. Fundamental considerations for the finite element analysis of shell structures. Comput. ES Structures, 66:19-36, 1998.

[7] D. Chapelle and K.J. Bathe. The mathematical shell model underlying general shell elements. Internat. J. Numer. Methods Engrg., 48(2):289-313, 2000.

[8] D. Chapelle and K.J. Bathe. The Finite Element Analysis of Shells - Fundamentals. Springer, second edition, 2011.

[9] D. Chapelle, A. Ferent, and K.J. Bathe. 3D-shell finite elements and their underlying model. Math. Models Methods Appl. Sci., 14(1):105-142, 2004.

[10] D. Chapelle, A. Ferent, and P. Le Tallec. The treatment of "pinching locking" in 3D-shell elements. ESAIM: M2AN, 37:143-158, 2003.

[11] P.G. Ciarlet. Mathematical Elasticity - Volume III: Theory of Shells. North-Holland, Amsterdam, 2000.

[12] M. Dauge and Z. Yosibash. Boundary layer realization in thin elastic 3D domains and 2D hierarchic plate models. Internat. J. Solids Structures, 37:2443-2471, 2000.

\footnotetext{
${ }^{1}$ See www.adina.com, in particular
} 
[13] M.C. Delfour. Intrinsic $P(2,1)$ thin shell model and Naghdi's models without a priori assumption on the stress tensor. In K.H. Hoffmann, G. Leugering, and F. Tröltzsch, editors, Optimal Control of Partial Differential Equations, pages 99-113, Basel, 1999. Birkhäuser.

[14] A.E. Green and W. Zerna. Theoretical Elasticity. Clarendon Press, Oxford, 2nd edition, 1968.

[15] D.N. Kim and K.J. Bathe. A 4-node 3D-shell element to model shell surface tractions and incompressible behavior. Comput. ES Structures, 86(21-22):2027-2041, 2008.

[16] W.T. Koiter. On the nonlinear theory of thin elastic shells. Proc. Kon. Ned. Akad. Wetensch., B69:1-54, 1965.

[17] J.L. Lions. Perturbations Singulières dans les Problèmes aux Limites et en Contrôle Optimal. Springer-Verlag, Berlin, New York, 1973.

[18] J.-C. Paumier and A. Raoult. Asymptotic consistency of the polynomial approximation in the linearized plate theory. ESAIM: Proc., 2:203-213, 1997.

[19] J. Pitkäranta, A.M. Matache, and C. Schwab. Fourier mode analysis of layers in shallow shell deformations. Comput. Methods Appl. Mech. Engrg., 190:2943-2975, 2001.

[20] A. Rössle, M. Bischoff, W. Wendland, and E. Ramm. On the mathematical foundation of the (1,1,2)-plate model. Int. J. Solids Struct., 36(14):2143-2168, 1999.

[21] J. Sanchez-Hubert and E. Sanchez-Palencia. Coques Elastiques Minces - Propriétés Asymptotiques. Masson, Paris, 1997. 